[Review]

\title{
基于碳材料的超级电容器电极材料的研究
}

\author{
李雪芹 ${ }^{1,2,8}$ 常 琳 $^{2,8} \quad$ 赵慎龙 $^{2} \quad$ 郝昌龙 $^{2} \quad$ 陆晨光 ${ }^{2, *} \quad$ 朱以华 ${ }^{1, *} \quad$ 唐智勇 ${ }^{2, *}$ \\ ('华东理工大学材料科学与工程学院, 超细材料制备与应用教育部重点实验室, 上海 200237; \\ 2 国家纳米科学中心，纳米系统与多级次制造重点实验室，北京 100190)
}

\begin{abstract}
摘要: 超级电容器作为一种新型的能源存储装置, 因为其比容量大、充放电速度快、循环寿命长等优点, 在储能领 域引起了极为广泛的关注。电极材料是决定超级电容器性能的核心因素, 其中, 常用的超级电容器电极材料主要有 如下三类: 碳基材料、金属氧化物及氢氧化物材料和导电聚合物材料。本文综述了超级电容器的工作原理并详细介 绍了基于碳材料及其二元、三元复合体系的电极材料的研究进展。
\end{abstract}

关键词: 超级电容器; 能量存储机理; 碳基材料; 电极材料

中图分类号: 0643

\section{Research on Carbon-Based Electrode Materials for Supercapacitors}

\author{
LI Xue-Qin ${ }^{1,2,8} \quad$ CHANG Lin ${ }^{2, \&} \quad$ ZHAO Shen-Long ${ }^{2} \quad$ HAO Chang-Long ${ }^{2}$ \\ LU Chen-Guang ${ }^{2, *} \quad$ ZHU Yi-Hua ${ }^{1, *} \quad$ TANG Zhi-Yong ${ }^{2, *}$
}

('Key Laboratory for Ultrafine Materials of Ministry of Education, School of Materials Science and Engineering, East China University of Science and Technology, Shanghai 200237, P. R. China; ${ }^{2}$ CAS Key Laboratory for Nanosystem and Hierarchy Fabrication, National Center for Nanoscience and Technology, Beijing 100190, P. R. China)

\begin{abstract}
As a new type of energy storage device, supercapacitors with high specific capacitance, fast charge and discharge, and long cycle life have attracted significant attention in the energy storage field. Electrode materials are a crucial factor defining the electrochemical performance of supercapacitors. The standard supercapacitor electrode materials used can be classified into three types: carbon-based materials, metal oxides and hydroxide materials, and conductive polymers. This review introduces the principles of supercapacitors and summarizes recent research progress of carbon-based electrode materials, including pure carbon materials, and the binary and ternary complex materials with carbon.
\end{abstract}

Key Words: Supercapacitor; Energy storage mechanism; Carbon material; Electrode material

\section{1 引 言}

随着工业的迅速发展, 人口的急剧增加, 全 球能源的消耗正以惊人的速度不断加快, 能源短
缺和环境污染成为当前人类发展面临的两大考 验。因此必须大力发展低成本、可持续并且环境 友好的新型能量转换和存储装置来满足现代社会

Received: June 13, 2016; Revised: August 31, 2016; Published online: September 1, 2016.

*Corresponding authors. ZHU Yi-Hua, Email: yhzhu@ecust.edu.cn; Tel: +86-21-64252022. LU Chen-Guang, Email: LUCG@ nanoctr.cn.

TANG Zhi-Yong, Email: zytang@nanoctr.cn; Tel: +86-10-82545659.

${ }^{\star}$ These authors contributed equally to this work.

The project was supported by the National Key Basic Research Program of China (973) (2014CB931801), National Natural Science Foundation of China (21676093, 21471056, 21473044, 21475029, 91427302), Instrument Developing Project of the Chinese Academy of Sciences (YZ201311), CAS-CSIRO Cooperative Research Program (GJHZ1503), and “Strategic Priority Research Program” of Chinese Academy of Sciences (XDA09040100). 国家重点基础研究发展规划(973)(2014CB931801), 国家自然科学基金(21676093, 21471056, 21473044, 21475029, 91427302), 中国科学院科研 装备研制项目(YZ201311), CAS-CSIRO合作研究项目(GJHZ1503)及中国科学院战略性先导科技专项(XDA09040100)资助

(C) Editorial office of Acta Physico-Chimica Sinica 
的发展需求和缓解日益突出的环境问题 ${ }^{1-4}$ 。在各 种储能系统中, 最主要的就是电化学能量存储系 统, 包括锂离子电池、超级电容器和燃料电 池 5,6 。近年来, 随着信息技术、电子产品和车用能 源等领域中新技术的迅速发展, 超级电容器凭借 着其比容量大、充放电速度快、循环寿命长等特 点吸引了研究者的极大兴趣。

超级电容器 $(\mathrm{SCs})$, 也称电化学电容器, 是介 于传统电容器和充电电池之间的一种新型、高

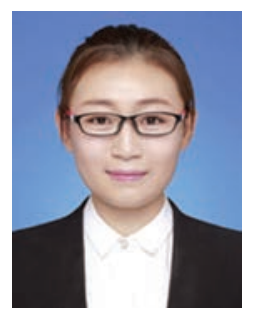

李雪芹, 本科毕业于青岛大学, 2014 年考入华东理工大学材料科 学与工程学院攻读硕士学位, 师从 朱以华教授, 期间于 2015 年 7 月至 今, 在国家纳米科学中心进行联合 培养。主要研究碳基材料在超级电 容器中的应用。
效、绿色的储能装置, 具有比普通静电电容器容 量大、比二次电池功率高、充放电速度快、循环 寿命长、工作温度范围宽、对环境无污染、无记 忆效应及免维护、安全性高等诸多优点, 在储能 领域得到广泛关注 7.8 。特别是近年来, 新能源汽车 的开发与应用, 促使人们对超级电容器技术提出 了更高的要求。图 1 所示的 Ragone 曲线图 ${ }^{9}$ 显示了 最重要的几种能量存储系统的功率密度和能量密 度之间的关系。在这些各种各样的能量存储系统 中, 锂离子电池(LIBs) 和超级电容器(SCs) 是两种已 经被广泛应用的技术体系。

$\mathrm{SCs}$ 的功率密度 $\left(10 \mathrm{~kW} \cdot \mathrm{kg}^{-1}\right)$ 高于 LIBs, 可以 在几秒钟内完全充电和放电, 完成大功率输入和 输出 ${ }^{10}$ 。它们在能量存储系统中起到补充或替换电 池的重要作用, 诸如用于不间断电源(储备电量用 于防止电力中断)、负荷调平等。近年来纳米材料 的广泛研究极大地推动了超级电容器等先进储能 技术的蓬勃发展。下面我们首先从超级电容器的

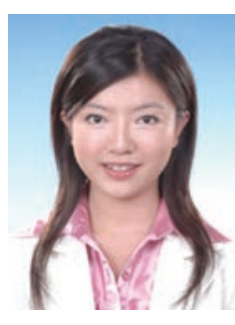

常琳, 工程师, 硕士毕业于北京工 业大学材料科学与工程专业, 研究 方向材料学。2010年 7 月硕士毕业 后加入国家纳米科学中心, 主要负 责 $X$ 射线衍射仪维护使用、技术培 训等工作。

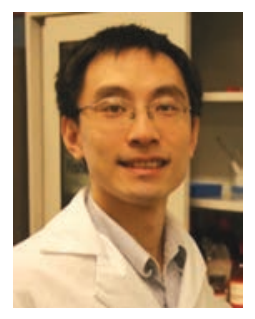

陆晨光, 本科毕业于北京大学化 学与分子工程学院, 美国 Duke大 学化学系理学博士。之后在美国 哥伦比亚大学应用物理系做博士 后研究员与助理研究员。2013 年 8 月至今, 在国家纳米科学中心任 副研究员。

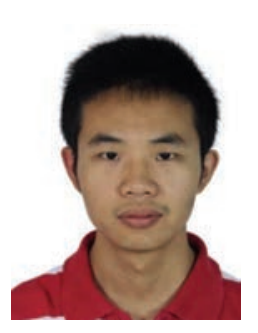

郝昌龙, 于 2015 年从江南大学获 得博士学位, 目前在国家纳米科学 中心进行博士后科研工作, 师从唐 智勇教授。研究工作主要集中于开 发低成本、新型高效的电化学催化 剂用于各种电化学过程, 例如超级 电容器、 $\mathrm{CO}_{2}$ 还原等。

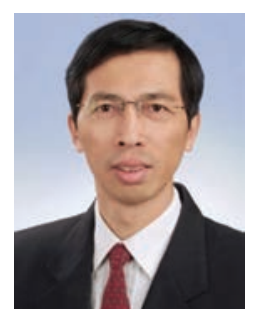

朱以华, 本科毕业于南京大学, 于 华东理工大学获得硕士和博士学 位。现在华东理工大学超细材料制 备与应用教育部重点实验室任教 授、博士生导师, 2015年当选英国 皇家化学会会士。主要研究纳米材 料的可控制备及在新能源中的应用。

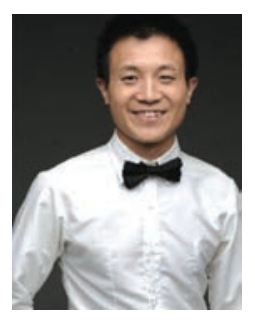

赵慎龙, 于 2011 年获得山东大学理 学学士学位。2013 年在哈尔滨工业 大学获得理学硕士学位。2013年至 今, 在哈尔滨工业大学攻读博士学 位, 师从唐智勇教授。主要研究金 属有机配位化合物材料的制备及其 在能源转化和电催化领域的应用。

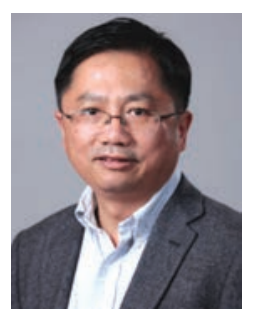

唐智勇, 理学博士, 研究员, 国家 杰出青年科学基金获得者, 科技部 纳米重大研究计划项目首席科学 家, 中国科学院纳米系统与多级次 制造重点实验室主任。研究领域为 纳米功能材料在环境和能源领域的 应用。 


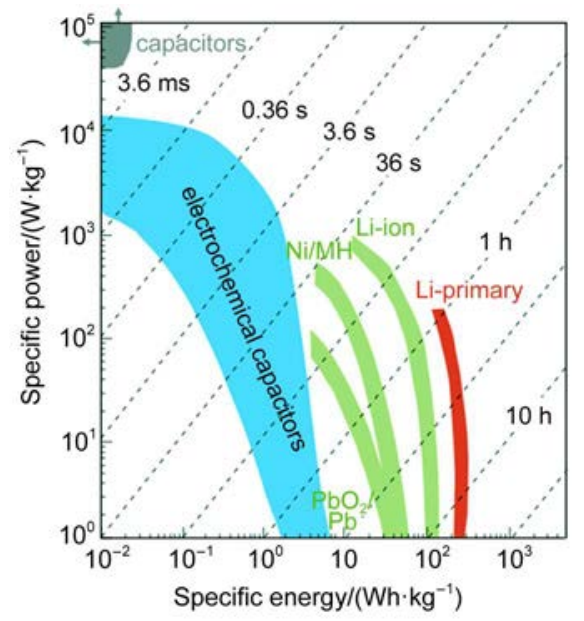

图 1 不同电化学能量储存装置的 Ragone曲线图9

Fig.1 Ragone plots for various electrochemical energy storage devices ${ }^{9}$

能量存储机理入手, 对其电极材料的研究进展进 行更深入的阐述。

\section{2 超级电容器的能量存储机理}

依据电荷存储机理及电极活性材料的不同, 可以将超级电容器分为双电层电容器、法拉第噟 电容器和混合型超级电容器, 如图 2 所示 ${ }^{11}$ 。

与传统的静电电容器电荷存储机理不同, 超 级电容器在高表面积的多孔电极材料与电解质溶 液的电化学界面处存储电荷。由于超级电容器电 极材料较高的比表面积以及电极与电解质离子更 短的距离, 使得超级电容器的比容量比传统的电 (a)

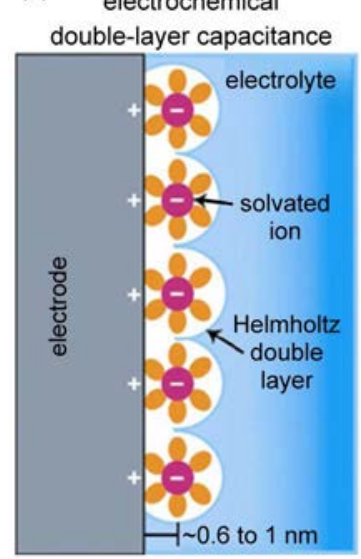

(b)

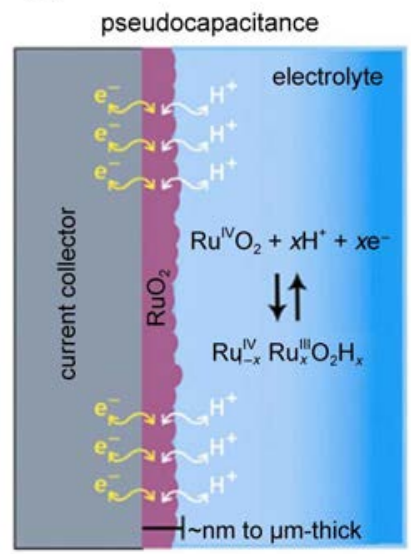

图 2 两种不同的电荷存储机理示意图 ${ }^{11}$

Fig.2 Scheme of two different charge storage mechanisms ${ }^{11}$

(a) electrochemical double-layer capacitance (EDLC); (b) redox reactions based pseudocapacitance
容器高几个数量级。超级电容器的比容量可以由 公式(1)进行描述:

$$
C=\frac{\varepsilon_{\mathrm{r}} \varepsilon_{0} A}{d}
$$

其中 $\varepsilon_{\mathrm{r}}$ 是电解质溶液的介电常数, $\varepsilon_{0}$ 是真空介电常 数, $d$ 是电解质离子与电极之间的距离, $A$ 是电极 材料的比容量。

\section{1 双电层电容器}

双电层电容器(EDLCs) 是目前研究最广泛、已 经商业化应用的一类超级电容器, 是基于正、负 离子在电极和电解液界面之间的表面上分别吸 附, 造成两个电极之间的电势差, 利用界面的双 电层电容来存储电荷从而达到能量存储的目的, 其储能机理简称为双电层理论(图 2(a))。

由于快速且近表面的电化学过程, EDLCs 可 以提供高的功率密度及优异的循环寿命。然而 EDLCs 存储的电能通常受限于在活性电极材料与 电解液之间有限的电荷分离以及由电解液可以稳 定的电势窗口决定的电势电压。EDLCs 的能量密 度可以由公式(2)计算得到:

$$
E=\frac{1}{2} C V^{2}
$$

其中 $C$ 是比容量, $V$ 是 EDLCs 的电势电压。由公式 (2)可以看出, $C 、 V$ 对 EDLCs 最终的性能都极其重 要。比容量在很大程度上取决于电极材料, 可以 通过选择高比表面积、高导电性的电极材料, 如 碳基的纳米材料 ${ }^{2}$ 来进行优化。对于两电极体系, 质量比容量可以由循环伏安曲线(公式(3))或恒电流 充-放电曲线(公式(4))计算得到:

$$
\begin{aligned}
& C=\frac{\int I \mathrm{~d} t}{m \Delta V v_{\mathrm{s}}} \\
& C=\frac{I \Delta t}{m \Delta V}
\end{aligned}
$$

其中 $m$ 为电极材料的负载质量, $I$ 为电流, $\Delta V$ 为电 势窗口, $v_{\mathrm{s}}$ 为电极扫描速率。另一方面, 对于电解 液的种类需要认真选取, 以得到最高的电势电 压。具有良好离子电导率的非水系电解液由于其 高达 3.5-4 V 的工作电势窗口常常被用在高能量密 度和高功率密度的 EDLCs 中; 对应的, 水系电解 液的电势窗口一般小于 $1.2 \mathrm{~V}$, 使其应用受到严重 的限制。根据公式(2)我们可以看出, 当电压 $V$ 增 加三倍时, 在相同条件下存储的能量 $E$ 增加了近一 个数量级。

基于碳基材料的各种有利特性, 双电层电容 
器的电极材料主要是具有高比表面积和高电导率 的碳材料 ${ }^{10,12}$, 包括活性炭 ${ }^{13}$ 、碳纳米管 ${ }^{14}$ 、石墨 烯 ${ }^{15}$ 以及其他纳米结构的碳材料等。

\section{2 法拉第赝电容器}

法拉第噟电容器利用电活性材料表面上发生 的快速、可逆的氧化还原反应来进行电荷存储(图 2(b))。法拉第电极的赝电容 $\left(300-1000 \mathrm{~F} \cdot \mathrm{g}^{-1}\right)$ 远大 于双电层存储机理的碳电极材料的比容量 $(100-$ $\left.250 \mathrm{~F} \cdot \mathrm{g}^{-1}\right)$ 。由于能量存储过程中涉及氧化还原反 应, 噟电容器可以达到较高的比容量和能量密 度, 但是与 EDLCs 相比会损失部分功率密度和循 环寿命。典型的噟电容活性材料包括过渡金属氧 化物, 如 $\mathrm{MnO}_{2}{ }^{16,17} 、 \mathrm{RuO}_{2}{ }^{18} 、 \mathrm{NiO}^{19} 、 \mathrm{Co}_{3} \mathrm{O}_{4}{ }^{20}$ 等, 导 电聚合物, 如聚吡咯、聚噻吩、聚苯胺及其它 $\pi$ 共轭的导电聚合物 ${ }^{21}$ 。图 3 比较了目前广泛研究的 一些最具代表性的超级电容器电极材料, 包括碳 基双电层电容器电极材料和法拉第赝电容器电极 材料 22 。

\section{3 混合型超级电容器}

除了EDLC 和法拉第噟电容器, 还有另外一种 特殊类型的电容器体系, 称为 “混合电容器”, 通 常是将一个电池型法拉第电极和一个电容电极结 合到一个超级电容器单元中。在该体系中, 电池 型电极提供高的能量密度, EDLCs 电极提供高的 功率密度。虽然这种类型的电容器与 EDLCs 相比 比容量和能量密度都有了极大的提高 ${ }^{23-25}$, 但是存 在一个致命的缺点, 法拉第电极的存在会降低混 合电容器整体的循环寿命 ${ }^{22}$ 。

综上所述, 电极材料的选择对提升超级电容
器功率密度、能量密度以及循环寿命是最为重要 的。其中, 合理设计各种材料之间的结合、形 貌、尺寸是改善和优化电极性能最有效的手段。

\section{3 电极材料}

超级电容器通常由电极、电解质、集流体、 隔膜及相应的辅助器件等部分组成, 其中起核心 作用的是电极材料, 决定着电容器的主要性能参 数, 也是对超级电容器的电化学性能进行改良和 优化的重要切入点。目前对于超级电容器的研究 工作主要集中在电极材料方面, 特别是开发具有 较高比容量且可在各种电解液中使用的电极材 料。常用的超级电容器电极材料主要有三类: 碳 基材料、金属氧化物及氢氧化物材料和导电聚合 物材料。这些电极材料都各有优缺点: 碳基材料 的循环稳定性好、工艺简单、成本低, 但比容量 低; 金属氧化物及氢氧化物材料的比容量大大高 于碳材料, 但成本较高、寿命短且有一定毒性; 导电聚合物材料具有良好的电子导电性、小内阻 及高比容量, 但在循环过程中容易发生体积膨胀 和收缩, 循环稳定性差。

\section{1 多孔碳材料}

\section{1 .1 活性炭}

活性炭具有高比表面积、低成本、化学稳定 性和热稳定性高以及电导率较高等优点, 在过去 的几十年中, 一直都是最常见的 EDLCs 电极材 料。活性炭一般是从各种类型的富含碳的有机前 驱体(椰子壳、木材、沥青、煤、聚合物等)在惰性 气氛中碳化得到的, 其中基于生物质的活性炭由

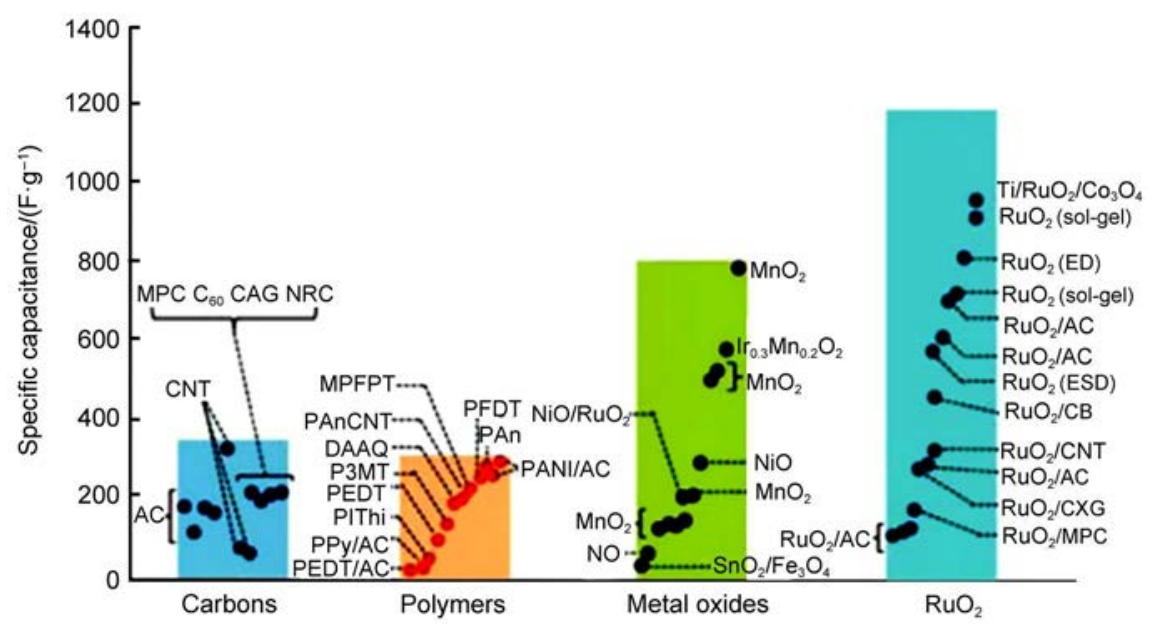

图 3 文献报道的 EDLC 和噟电容器电极材料的电容性能 ${ }^{22}$

Fig.3 Capacitive performance of various electrode materials reported in the literature ${ }^{22}$ 
于来源广, 吸引了科学家们极大的关注。碳化后 的活性炭往往会伴随有物理或化学活化作用, 与 没有经活化处理的活性炭相比, 比表面积和孔体 积均会显著提高。活化过程和所使用的碳前驱体 不同, 所制得的活性炭的物理化学性质和比表面 积也会不同, 目前所制得的活性炭材料比表面积 可以达到 $3969 \mathrm{~m}^{2} \cdot \mathrm{g}^{-126}$ 。

水热法碳化是目前日益流行的一种由生物质 材料制备多孔碳材料的方法, 具有成本低, 所需 温度低, 合成过程中不需要使用有毒化学试剂、 环境友好等优点。用水热法碳化纤维素、马铃 薯、锯末等材料并用氢氧化钾进行活化, 所得的 活性炭比表面积达 2125-2967 $\mathrm{m}^{2} \cdot \mathrm{g}^{-1}$ 。通过水热法 获得的活性炭, 虽然表面积会适度增加, 但几乎 不存在开放的孔隙, 严重阻碍了EDLCs 在电动汽 车等领域中的应用 ${ }^{27}$ 。

目前各种合成的聚合物, 如苯酚甲醛树脂、 聚苯乙烯树脂、聚糠醇、聚苯胺以及聚吡咯等, 具有成本低、商业实用性、结构均匀以及化学耐 受性强等优点, 可作为替代的前驱体。Yan 等 ${ }^{28}$ 由 棒状的聚苯胺碳化并用 $\mathrm{KOH}$ 活化制得活性炭, 由 于 $1976 \mathrm{~m}^{2} \cdot \mathrm{g}^{-1}$ 的高表面积、窄的孔径分布 $(<3 \mathrm{~nm})$ 以及短的扩散长度, 其显示出较高的比容量 $(455$ $\left.\mathrm{F} \cdot \mathrm{g}^{-1}\right)$ 和显著的倍率性能。Wei 等 ${ }^{29}$ 用聚吡咯经一步 法用 $\mathrm{KOH}$ 活化制备活性炭, 所制备的碳材料具有 $2.39 \mathrm{~cm}^{3} \cdot \mathrm{g}^{-1}$ 的孔体积和高达 $3432 \mathrm{~m}^{2} \cdot \mathrm{g}^{-1}$ 的比表面 积。应用于 $\mathrm{EDLCs}$, 在 $60{ }^{\circ} \mathrm{C}$ 的 $\mathrm{IL} \mathrm{EMImBF}_{4}$ 离子 液体中产生了高达 $300 \mathrm{~F} \cdot \mathrm{g}^{-1}$ 的高比容量。

迄今为止, 由各种前驱体通过物理或化学活 化制备的活性炭是商业超级电容器中使用最广泛 的电极材料。然而, 由于能量密度比较低、孔结 构的难以控制, 活性炭的实际应用仍被限定于一 定的范围内, 如何扩大其应用范围仍然是一个很 大的挑战。

\section{1 .2 模板碳}

大多数多孔碳材料具有非常宽的孔径分布与 随意连接的孔道, 及无序和复杂的结构, 导致材 料的导电性和离子传输差, 使得在超级电容器的应 用中倍率性能受到一定的限制。此外, 在纳米尺度 下精确地控制传统碳材料的结构非常困难。因此, 模板法被认为是一种能够提供良好设计和精确地控 制碳材料的有效、独特且多功能的方式 ${ }^{30}$ 。

利用模板法制备的碳材料(模板碳)表现出相对
活性炭较低的比表面积、较高的孔隙率、可控的 孔径分布以及相互联通的孔隙网络。到目前为 止, 模板法合成纳米结构的碳材料已经取得了显 著的进展。通常, 模板碳的合成过程会涉及以下 几个步骤: 首先碳前驱体(如蔗糖、丙烯、糠醇、 酚醛树脂或聚合物溶液)浸渍到多孔结构的模板 中, 随后进行碳化处理, 最后除去模板得到多孔 碳材料。根据使用的模板不同, 模板法可分为硬 模板法和软模板法。前者是指硬模板的复制合 成, 通过渗透、碳化并除去预合成的硬模板。各 种无机材料, 例如二氧化硅纳米颗粒、沸石、阳 极氧化铝( $\mathrm{AAO}$ )膜、介孔二氧化硅、 $\mathrm{CaCO}_{3} 、 \mathrm{MgO}$ 等都可被用作硬模板。而后者为软模板的自组 装, 经缩合和碳化过程得到多孔碳材料, 且模板 无需除去。近年来各种市售的三嵌段共聚物, 如 P123、F127、F108广泛被用作软模板剂。取决于 使用的模板和碳前驱体的不同, 模板法可制备各 种具有不同结构的微孔、介孔、大孔碳材料。

金属-有机骨架配位化合物(MOFs)，也称多孔 配位聚合物(PCPs), 是一种有机-无机杂化的多孔 材料, 由含氧、氮的多齿有机配体与金属原子或 金属原子簇以配位共价键相连接, 通过自组装形 成的具有周期性网络结构的类沸石材料 ${ }^{31,32}$ 。基于 有机连接配体的几何形状和与无机金属离子或金 属离子团簇的相互协调作用, 它们的结构可以根 据目标性能进行设计 ${ }^{33-35}$ 。MOFs 材料的主要结构 特征是超高的孔隙率(高达 $90 \%$ 的自由体积), 超大 的内部表面积, 伸展开的 Langmuir 表面积超过 $10000 \mathrm{~m}^{2} \cdot \mathrm{g}^{-136-38}$, 还具有可调控的孔径尺寸、可修 饰的孔道表面, 超低的密度等独特性能优势, 使 MOFs 广泛应用于气体储存和分离 ${ }^{39,40}$ 、催化 ${ }^{41,42}$ 、 化学传感器 ${ }^{43,44}$ 、质子传导 ${ }^{45}$ 和药物传输 ${ }^{46}$ 等领域。 MOFs 作为一类新型的多孔材料, 具有永久的纳米 空腔和开放的通道, 为小分子的进出提供了良好的 先天条件。近年来, 以 MOFs 为模板合成纳米多孔 碳材料成为一大研究热点 ${ }^{47,48}$, 在此重点进行介绍。

目前 MOFs 作为前驱体碳化制备多孔碳材料主 要有两种方法, 如图 4 所示 ${ }^{49}$, 第一种是 MOFs 作 为单一碳源直接在特定的气氛中(氮气、氩气等)进 行高温煅烧碳化; 第二种是 MOFs 和其他小分子有 机物, 如糠醇、葡萄糖、乙二胺、四氯化碳、酚 醛树脂等 ${ }^{50-52}$ 共同作为碳源在一定气氛下 $($ 氮气、氩 气等)高温炦烧, 这些有机物通常首先通过浸渍法 

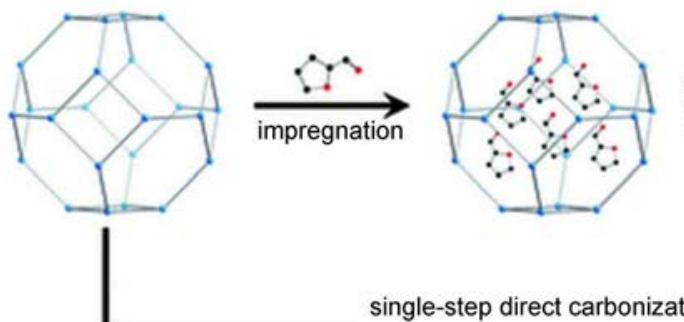

$\stackrel{\text { polymerization }}{\longrightarrow}$

$\overrightarrow{\text { carbonization }}$

single-step direct carbonization

图 4 MOFs 通过两步煅烧法和一步直接煅烧法制备纳米多孔材料示意图 ${ }^{49}$

Fig.4 Schematic representation of construction of nanoporous materials from MOFs with two-step method and direct carbonization of $\mathrm{MOFs}^{49}$

或气相沉积法等进入 MOFs 的孔道内, 随后在 MOFs 的孔道内进行聚合。在碳化过程中, 外加的 有机碳源有助于得到金属粒子均匀分散的纳米多 孔碳材料。这两种方法对于制备特定纳米结构和 功能的多孔碳材料各有优缺点。

用于制备多孔碳材料比较代表性的 MOFs 主要 有 MOF-5, Al 基多孔配位聚合物(Al-PCP)和 ZIF-8 等 ${ }^{49}$ 。2008 年, Xu 课题组 47 首次报道了用 MOF-5 作为牺牲模板与外加的碳源共同作为前驱体煅烧 碳化制得了多孔碳材料。如图 5 所示, 糠醇(FA)蒸 汽通过气相沉积法进入 MOF-5 的孔道, 并在孔道 内进行聚合, 随后将材料在氩气中进行煅烧碳化 制备得到多孔碳材料。研究表明, 经高温碳化制 备的纳米多孔碳材料 (NPC) 的 BET 比表面积高达 $2872 \mathrm{~m}^{2} \cdot \mathrm{g}^{-1}$, 具有很好的 $\mathrm{H}_{2}$ 吸附效果; 用作双电 层电容器的电极材料同样表现出很好的电化学性 能, 以 $1 \mathrm{~mol} \cdot \mathrm{L}^{-1} \mathrm{H}_{2} \mathrm{SO}_{4}$ 做电解液, 扫描速率为 5 $\mathrm{mV} \cdot \mathrm{s}^{-1}$ 时的比容量高达 $204 \mathrm{~F} \cdot \mathrm{g}^{-1}$ 。基于以上研 究, Liu 等 ${ }^{50}$ 又深入研究了碳化温度对多孔碳材料

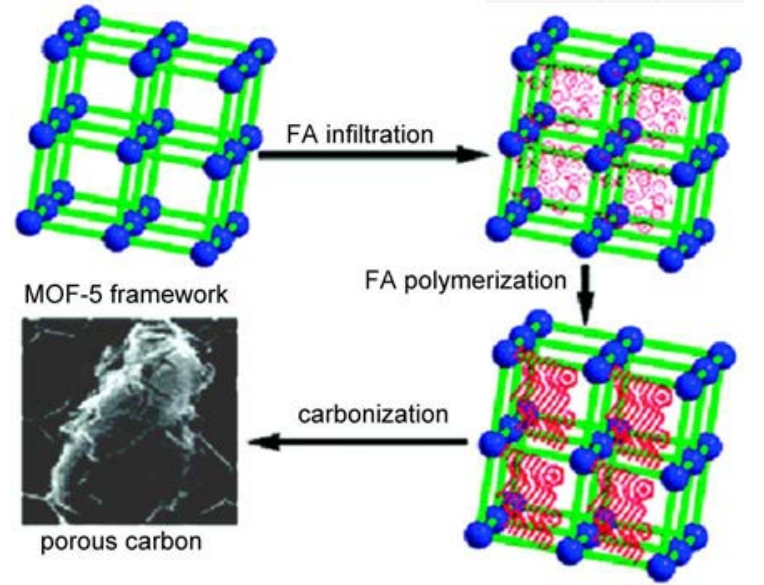

图 5 MOF-5 与 FA 碳化制备多孔碳的示意图 ${ }^{47}$

Fig.5 Schematic illustration of the carbonization procedure of MOF-5 infiltrated with FA precursors ${ }^{47}$
孔结构的影响。通过蒸汽沉积法使糠醇进入 MOF5 孔道内共同作为碳源, 在 $530-1000{ }^{\circ} \mathrm{C}$ 的温度下 煅烧, 得到多孔碳材料的比表面积为 1141-3040 $\mathrm{m}^{2} \cdot \mathrm{g}^{-1}$, BET 表面积随碳化温度的变化呈现出 “V” 字形的趋势, 所有 5 个 NPC 样品的孔径分布 集中在大约 $3.9 \mathrm{~nm}$ 。可以看出, 碳化温度的选择对 多孔碳材料的比表面积起到了至关重要的作用。

2011 年, Jiang 等 ${ }^{53}$ 使用 ZIF-8 同时作为前驱体 和模板, 以糠醇 $(\mathrm{FA})$ 作为外加碳源制备纳米多孔碳 材料。通过改变抜烧温度 $800-1000{ }^{\circ} \mathrm{C}$, 得到碳材 料的表面积可以调整从 $2169-3405 \mathrm{~m}^{2} \cdot \mathrm{g}^{-1}$ 。作为参 照, ZIF-8 在 $1000{ }^{\circ} \mathrm{C}$ 直接碳化得到了表面积高达 $3184 \mathrm{~m}^{2} \cdot \mathrm{g}^{-1}$ 的多孔碳材料。这类高度多孔的碳材 料表现出比大多数的 MOF 前驱体高得多的储氢容 量。循环伏安 $(\mathrm{CV})$ 测量结果表明, 基于这两种碳 材料的电极都显示出规则的矩形形状, 没有任何 氧化还原峰。恒电流充-放电测量得到在 $50 \mathrm{~mA} \cdot \mathrm{g}^{-1}$ 的电流密度下的比容量约为 $200 \mathrm{~F} \cdot \mathrm{g}^{-1}$ 。2012 年, Yamauchi 课题组 ${ }^{54}$ 在 $800{ }^{\circ} \mathrm{C}$ 下直接碳化 $\mathrm{Al}-\mathrm{PCP}$, 所得纳米多孔碳材料比表面积高达 $5000 \mathrm{~m}^{2} \cdot \mathrm{g}^{-1}$, 并利用石英晶体微天平技术测试了其对有毒的芳 烃类物质的感测能力, 结果显示 PCP-800 可以吸收 大量的苯蒸汽并能在几秒后快速作出响应。

最近, Yamauchi 等 ${ }^{55}$ 通过核-壳结构的 MOFs 作为前驱体, 得到了一种选择性功能化的 $\mathrm{N}$ 掺杂 的多孔碳材料, 如图6 所示。由ZIF-8@ZIF-67 晶 体作为前驱体, 在 $\mathrm{N}_{2}$ 中䍩烧 $3 \mathrm{~h}$, 得到一种石墨烯 碳包覆多孔 $\mathrm{N}$ 掺杂碳 $(\mathrm{NC} @ \mathrm{GC})$ 的核- 壳结构材料, 其中 $\mathrm{N}$ 掺杂的多孔碳作为核, 高度石墨化的碳作 为壳。 $\mathrm{NC} @ \mathrm{GC}$ 融合了 $\mathrm{NC}$ 和 $\mathrm{GC}$ 各自的优点, 如 高表面积、高的 $\mathrm{N}$ 含量、石墨烯化结构等, 同时 还具有由ZIF-8@ZIF-67 带来的相互连通的多级次 的微孔/介孔结构, 这对该材料应用于超级电容器 


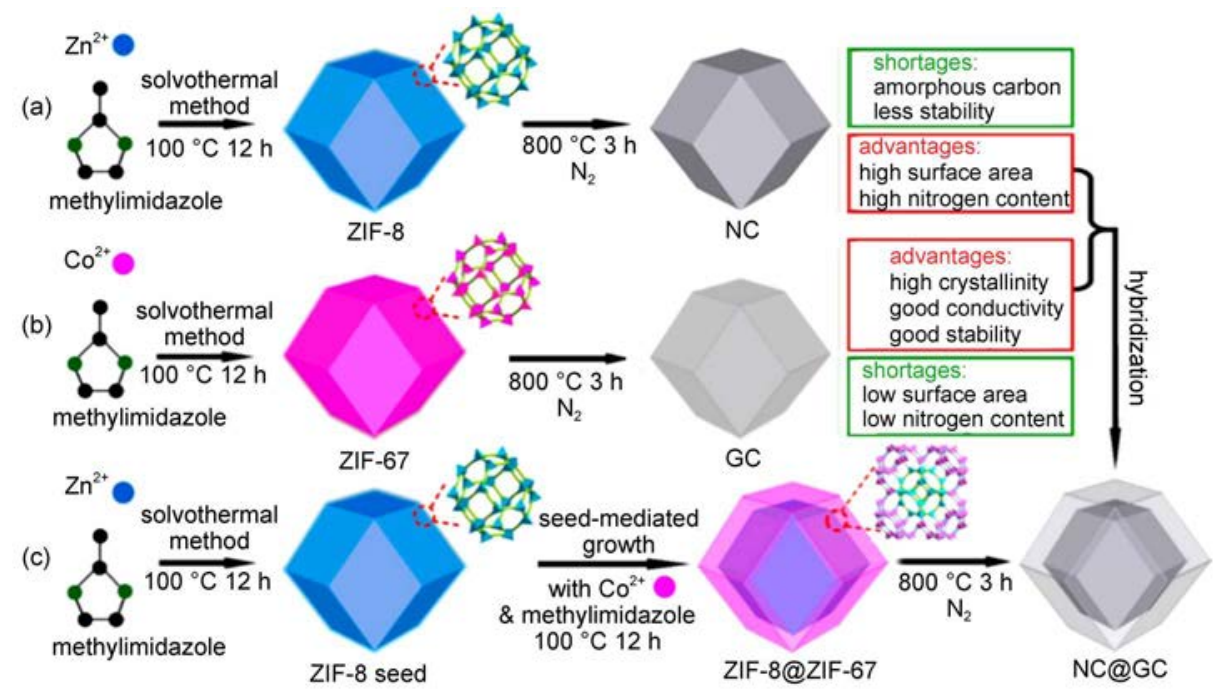

图6ZZIF-8@ZIF-67 的制备示意图 ${ }^{55}$

Fig.6 Synthetic scheme for the preparation of ZIF-8@ZIF-67 ${ }^{55}$

(a) ZIF-8 crystals and NC, (b) ZIF-67 crystals and GC, (c) core-shell ZIF-8@ZIF-67 crystals and NC@GC

以及氧还原反应 $(O R R) 、 L I B s$ 等其他领域都是非常 有利的。由恒电流充放电曲线计算得到的在电流 密度为 $2 \mathrm{~A} \cdot \mathrm{g}^{-1}$ 时的比容量高达 $270 \mathrm{~F} \cdot \mathrm{g}^{-1}$ 。

\subsection{3 碳纳米管(CNTs)}

碳纳米管 $(\mathrm{CNTs})$ 可看作是石墨烯片层卷曲而 成, 按石墨烯片层数的不同可将 CNTs 分为单壁碳 纳米管 $(\mathrm{SWNTs})$ 和多壁碳纳米管 $(\mathrm{MWNTs})^{56}$ 。CNTs 具有独特的中空结构、良好的导电性、高的可接 触表面积、稳定性好以及交互缠绕的纳米大小网 状结构等优良特性 ${ }^{56-60}$ 。1997 年, Niu 等 ${ }^{59}$ 首先将 CNTs 用作超级电容器电极材料, 随后由于其独特 的结构和机械性能引起广泛关注, 被认为是高功 率超级电容器的理想电极材料。

CNTs 的孔径绝大部分在 $2 \mathrm{~nm}$ 以上, 有利于双 电层的形成, 但由于 CNTs 的比表面积较低 $(<600$ $\left.\mathrm{m}^{2} \cdot \mathrm{g}^{-1}\right)^{61}$, 以 CNTs 作为超级电容器电极材料的比 电容通常 $<100 \mathrm{~F} \cdot \mathrm{g}^{-1}$, 且存在充放电效率较低、自 放电现象严重、易发生团聚等现象, 不能很好地 满足实际需要 ${ }^{2}$ 。所以需要对 CNTs 进行处理, 提 高其电化学性能。

在过去的几年中, 为满足用于便携式电子设 备, 包括卷曲显示器, 伸缩性集成电路, 和用于 个人多媒体或医疗设备可穿戴系统的需求, 柔性 和轻量的能量存储系统引起广泛的研究兴趣 ${ }^{63,64}$ 。 将碳纳米管印在塑料、纸上或涂在纺织品上制作 薄且柔软的超级电容器 ${ }^{63-68}$, 在这些电极上, 碳纳 米管不仅充当高导电性和柔性的活性物质, 同时
也增加膜的有效接触表面积, 使薄膜超级电容器 的效率最大化。

单壁碳纳米管涂覆塑料作为两个电极和集流 体制成的薄膜超级电容器, 在有机电解质中表现 出较高的能量密度 $\left(6 \mathrm{Wh} \cdot \mathrm{kg}^{-1}\right)$ 和功率密度 $(70 \mathrm{~kW} \cdot$ $\left.\mathrm{kg}^{-1}\right)^{66}$ 。用大规模、独立、灵活的单壁碳纳米管薄 膜作为阳极和阴极制造的紧凑设计的超级电容 器, 由于内阻小, 表现出很高的能量密度( 43.7 $\left.\mathrm{Wh} \cdot \mathrm{kg}^{-1}\right)$ 和功率密度 $\left(\sim 193.7 \mathrm{~kW} \cdot \mathrm{kg}^{-1}\right)$ (图 7(a) $)^{65}$ 。 使用单壁碳纳米管涂覆的织物作为超级电容器的 电极, 制备了可拉伸、耐磨的超级电容器 (图 7 (b)), 在 $1 \mathrm{~mol} \cdot \mathrm{L}^{-1}$ 的 $\mathrm{LiPF}_{6}$ 电解质中显示出了 $140 \mathrm{~F}$. $\mathrm{g}^{-1}$ 的高比容量以及 $20 \mathrm{Wh} \cdot \mathrm{kg}^{-1}$ 的能量密度和 10 $\mathrm{kW} \cdot \mathrm{kg}^{-1}$ 的功率密度。此外, 还表现出极佳的循环 稳定性, 130000 次循环后只有 $2 \%$ 的电容损失 ${ }^{68}$ 。

为增加 CNTs 表面的亲水性, 通常对 CNTs 进 行酸、碱活化处理、热氧化处理 ${ }^{69}$ 和电化学氧化处 理等措施, 这不仅提高了 CNTs 的纳米结构的有序 性, 还有效改善了 CNTs 的电容性能并增加循环寿 命。高度有序排列的碳纳米管阵列与自由生长的 CNTs 相比, 具有更大的比容量和更好的倍率性 能 ${ }^{70}$, 制备碳纳米管阵列已引起人们的广泛关 注 $^{71}$ 。此外, 可将 CNTs 和金属氧化物或导电聚合 物复合, 提高材料的能量密度, 从而得到低成 本、高性能的复合电极材料。

\section{1 .4 石墨烯}

石墨烯是从石墨材料中剥离出来, 由碳原子 


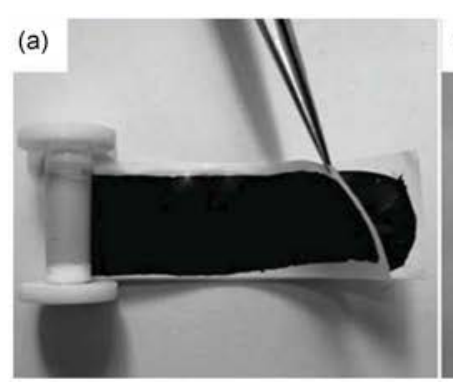

(b)

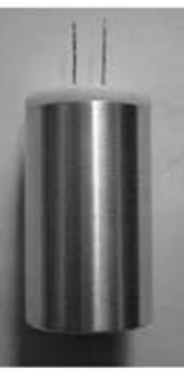

(c)

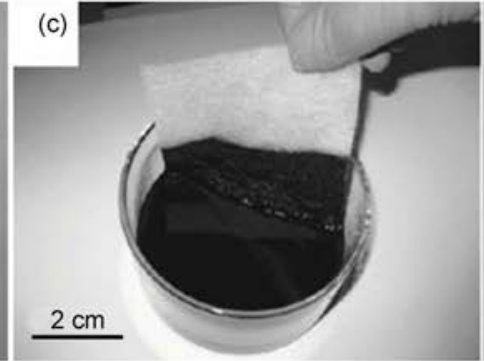

(c)

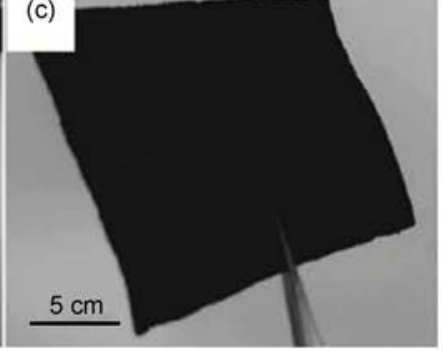

图 7 碳纳米管在超级电容器电极材料中的应用

Fig.7 Carbon nanotubes applied in supercapacitor electrode materials

(a) rolled design of the separator with SWNT films and (b) the resulting compact-designed supercapacitor ${ }^{65}$;

(c) conductive textiles are fabricated by dipping textile into an aqueous SWNT ink followed by drying in oven at $120{ }^{\circ} \mathrm{C}$ for $10 \mathrm{~min}$;

(d) a thin, $10 \mathrm{~cm} \times 10 \mathrm{~cm}$ textile conductor based on a fabric sheet with $100 \%$ cotton $^{68}$

组成的只有一层原子厚度的二维晶体。2004 年, Novoselov 和 Geim ${ }^{72}$ 用胶带从块体石墨中剥离出了 单层的石墨烯, 从而引发了大量对石墨烯的研 究。石墨烯具有很多独特的性质, 包括理论比表 面积大 $\left(2630 \mathrm{~m}^{2} \cdot \mathrm{g}^{-1}\right)^{73}$ 、导电性好 ${ }^{72}$ 、化学稳定性 和热稳定性好 ${ }^{74}$ 、电势窗口宽 ${ }^{75}$ 以及柔性好 ${ }^{76}$ 等。 石墨烯基的材料广泛用于制备柔性超级电容器的 电极材料 ${ }^{72,73,77-80}$ 。测量得到的单层石墨烯的电化学 双层电容约为 $21 \mu \mathrm{F} \cdot \mathrm{cm}^{-2}$, 因此, 如果它的整个表 面区域可被充分利用, 基于石墨烯的超级电容器 能够实现高达 $550 \mathrm{~F} \cdot \mathrm{g}^{-1}$ 的理论电化学双层电容, 是所有碳材料比容量的上限。

2013 年, $Y u$ 课题组 ${ }^{81}$ 用水热法以有机胺和氧 化石墨烯合成了 $\mathrm{N}$ 掺杂的石墨烯水凝胶 $(\mathrm{GN}-\mathrm{GH})$, 其中有机胺作为 $\mathrm{N}$ 源的同时还对石墨烯片层的组 装起调控作用, 从而调控所得到的三维材料的内 部结构。所得到的 GN-GH 用作超级电容器的电极 材料时, 超级电容器的性能得到显著提高, 即使 在 $185 \mathrm{~A} \cdot \mathrm{g}^{-1}$ 的超快充放电速率下, 比容量仍能达 到 $113.8 \mathrm{~F} \cdot \mathrm{g}^{-1}$, 功率密度达 $205.0 \mathrm{~kW} \cdot \mathrm{kg}^{-1}$ 。此 外, 电流密度为 $100 \mathrm{~A} \cdot \mathrm{g}^{-1}$ 时, 经历 4000 次循环后 的比容量损失为 $4.8 \%$, 如图 8 所示。

同年, Duan 课题组 ${ }^{82}$ 报道了用对苯二酚一步 法还原氧化石墨烯制备功能化的石墨烯凝胶 (FGHs), 如图 9 所示。FGHs 具有很好的机械强 度, 可以直接用来作为超级电容器的电极材料而 不用额外加入其他的粘结剂和导电物质。在 $1 \mathrm{~mol}$ $\mathrm{L}^{-1} \mathrm{H}_{2} \mathrm{SO}_{4}$ 电解液中, 电流密度为 $1 \mathrm{~A} \cdot \mathrm{g}^{-1}$ 时的比容 量达到 $441 \mathrm{~F} \cdot \mathrm{g}^{-1}$, 比未经功能化的石墨烯水凝胶 的 $221 \mathrm{~F} \cdot \mathrm{g}^{-1}$ 高两倍。同时, 该材料也表现出了很 好的速率性能和循环稳定性。以 FGHs 为电极材
料, $\mathrm{H}_{2} \mathrm{SO}_{4}$ - $\mathrm{PVA}$ 凝胶作为电解质组成的柔性超级电 容器, 不但能实现近似单电极在水系电解液中的 电容性能, 还具有很好的柔性、低自放电性。

最近, Huang 等 ${ }^{83}$ 设计合成了一种氮掺杂的有 序介孔石墨烯, 如图 10 所示, 具有优异的电化学 储能特性, 比容量高达 $855 \mathrm{~F} \cdot \mathrm{g}^{-1}$, 利用该材料组 装成的对称超级电容器能够进行快速的充放电, 在水性电解液中工作时的能量密度达到 $41 \mathrm{Wh}$. $\mathrm{kg}^{-1}$, 功率密度达到 $26 \mathrm{~kW} \cdot \mathrm{kg}^{-1}$, 性能不亚于商用 的碳基电容器。这种高性能主要起源于 $\mathrm{N}$ 掺杂诱 生了氧化还原反应, 在不降低材料高导电性的前 提下, 增加了电化学储能活性。

超级电容器可以通过将纳米结构的碳材料与 噟电容材料进行复合, 在纳米结构的杂化复合材 料电极中引入噟电容作用来提高其电化学性能, 例如将过渡金属氧化物与导电聚合物复合 ${ }^{84}$ 。为改 善金属氧化物电极材料低电导率导致的容量低、 循环寿命短等问题, 最常采用的方法之一就是与 高导电性的材料进行复合。

\section{2 二元体系复合电极}

\subsection{1 碳材料-金属氧化物}

\subsubsection{1 $\mathrm{RuO}_{2}-$ 碳材料}

由于 $\mathrm{RuO}_{2}$ 具有很高的比容量而得到广泛重 视, 研究主要集中在使用各种方法制备大比表面 积的 $\mathrm{RuO}_{2}$ 。将 $\mathrm{RuO}_{2}$ 与各种碳材料进行结合不但可 以减少贵金属材料的用量, 降低成本, 同时也能 很好地利用碳材料的高比表面积、高导电性等优 异特性提高材料的比容量。

Wang 等 ${ }^{85}$ 把 $\mathrm{RuCl}_{3} \cdot x \mathrm{H}_{2} \mathrm{O}$ 溶于无水乙醇中, 加 入活性炭并分散均匀, 滴加 $\mathrm{KOH}$ 水溶液反应制备 了 $\mathrm{RuO}_{2} / \mathrm{AC}$ 复合材料。以 $1 \mathrm{~mol} \cdot \mathrm{L}^{-1} \mathrm{KOH}$ 为电解 

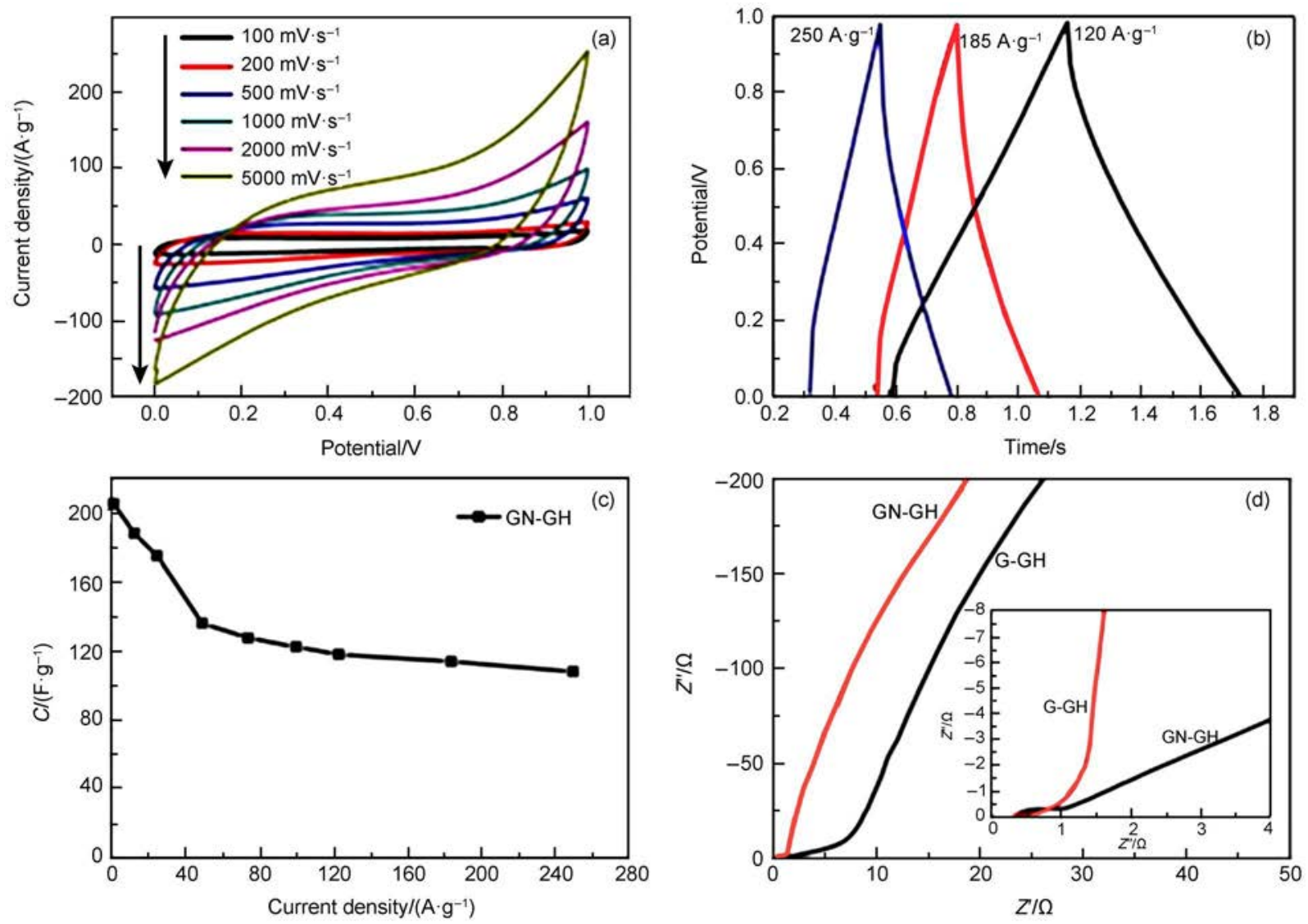

图 8 GN-GH 的超级电容器性能 ${ }^{81}$

Fig.8 Supercapacitor performance of the typical GN-GH ${ }^{81}$

(a) cycle voltammetry (CV) curves for different scan rates; (b) Galvanostatic charge/discharge curves of the GN-GH supercapacitor under different constant currents; (c) plot of specific capacitance versus discharging current density; (d) Nyquist plots of a typical GN-GH and G-GH supercapacitors

液，当电流密度为 $20 \mathrm{~mA} \cdot \mathrm{cm}^{-1}$ 时，炭含量为 $60 \%$ 的复合材料的比容量高达 $359 \mathrm{~F} \cdot \mathrm{g}^{-1}$, 远高于单一 成分活性炭材料的比容量 $\left(160 \mathrm{~F} \cdot \mathrm{g}^{-1}\right)$ 。将 $\mathrm{RuO}_{2}$ 与导 电性更高的碳材料进行结合, 从而提高整体材料 导电性, 在更大程度上提高性能。 $\mathrm{Wu}$ 等 ${ }^{86}$ 制备得 到 $\mathrm{RGO} / \mathrm{RuO}_{2}$ 复合材料表现出较高的比容量( 570 $\mathrm{F} \cdot \mathrm{g}^{-1}, \mathrm{Ru}$ 的负载量为 $\left.38.3 \%\right)$, 良好的稳定性(1000 循环后的比容量损失 $2.1 \%)$, 以及高能量密度 $\left(20.1 \mathrm{Wh} \cdot \mathrm{kg}^{-1}\right)$ 和功率密度 $\left(10 \mathrm{~kW} \cdot \mathrm{kg}^{-1}\right)$ 。实验证 明, 高度分散的 $\mathrm{RuO}_{2}$ 纳米粒子由于尺寸的减小使 得内部的 $\mathrm{RuO}_{2}$ 也可以接触到电解质离子, 从而在 很大程度上提高了比容量。另一方面, 导电的碳材 料基底大大增强了 $\mathrm{RuO}_{2}$ 的导电性，有利于离子的 渗透, 大幅缩短离子运输距离。又如, $\mathrm{Shu}$ 等 ${ }^{87}$ 将 $\mathrm{RuO}_{2} \cdot n \mathrm{H}_{2} \mathrm{O}$ 与 MWNT 复合得到的 $\mathrm{RuO}_{2} \cdot n \mathrm{H}_{2} \mathrm{O}$ / MWNT 电极材料, 在 $\mathrm{H}_{2} \mathrm{SO}_{4}$ 水系电解液中的比容 量高达 $1652 \mathrm{~F} \cdot \mathrm{g}^{-1}$ 。近年来, 为降低材料的成本, 研究者们一直在积极研究寻找相对价格低廉、环
境友好又有相似电化学行为的替代材料, 如 $\mathrm{MnO}_{2} 、 \mathrm{NiO} 、 \mathrm{Co}_{3} \mathrm{O}_{4} 、 \mathrm{~V}_{2} \mathrm{O}_{5}$ 等。

\subsubsection{2 $\mathrm{MnO}_{2}$-碳材料}

$\mathrm{MnO}_{2}$ 具有 $1370 \mathrm{~F} \cdot \mathrm{g}^{-1}$ 的高理论比容量、低成 本以及环境友好等特性 ${ }^{8}$, 是作为超级电容器噟电 容电极材料最具潜力的过渡金属氧化物, 吸引了 广泛的关注 ${ }^{89} 。 \mathrm{MnO}_{2}$ 提供赝电容的电荷存储机理 是基于 $\mathrm{MnO}_{2}$ 纳米材料表面或近表面 $\mathrm{III} / \mathrm{IV}$ 氧化价 态的改变, 反应如公式(5):

$$
\mathrm{MnO}_{2}+x \mathrm{C}^{+}+y \mathrm{H}^{+}+(x+y) \mathrm{e}^{-} \rightleftharpoons \mathrm{MnOOC}_{x} \mathrm{H}_{y}
$$

式中 $\mathrm{C}^{+}$代表电解质阳离子, 如 $\mathrm{H}^{+} 、 \mathrm{~K}^{+} 、 \mathrm{Na}^{+} 、 \mathrm{Li}^{+}$ 等 ${ }^{90,91}$ 。然而, 在实际的实验中 $\mathrm{MnO}_{2}$ 的理论容量不 可能达到, 主要是由于其较差的电子传导性限制 了高功率性能所需的速率性能, 从而阻碍在能量 存储系统中的实际应用。为了提高 $\mathrm{MnO}_{2}$ 基电极材 料的导电性得到高的比容量, 大量的研究集中在 探索 $\mathrm{MnO}_{2}$ 与其他高电导率的材料如碳纳米材料、 金属纳米结构的材料等进行复合, 以弥补 $\mathrm{MnO}_{2}$ 较 

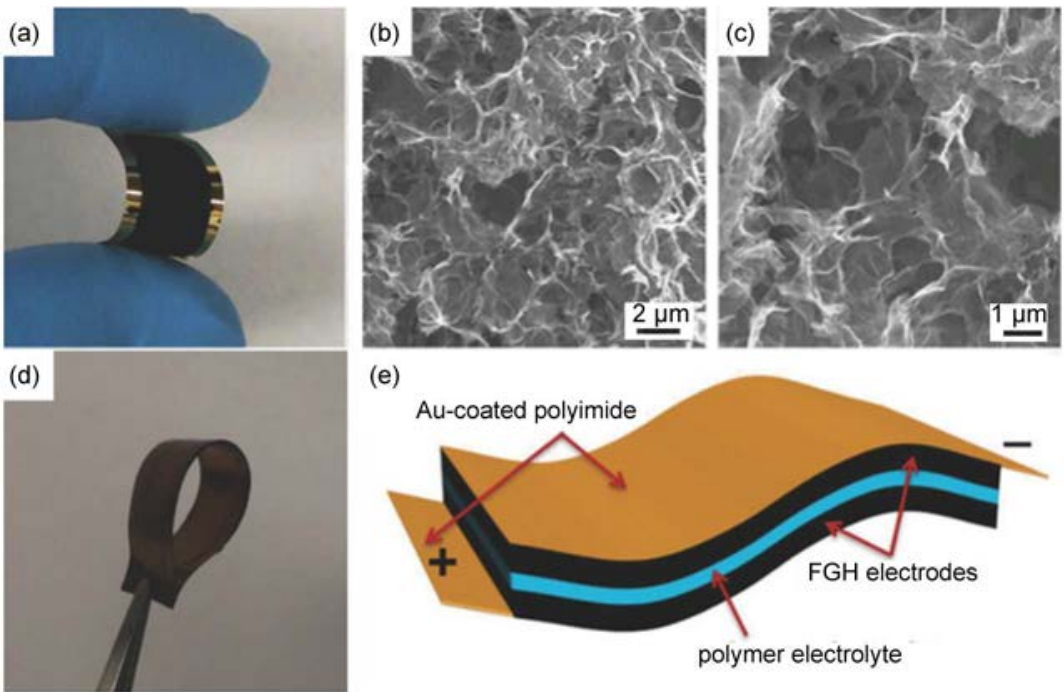

图9 FGH 柔性薄膜电极 ${ }^{82}$

Fig.9 Flexible FGH thin film electrodes ${ }^{82}$

(a) digital photograph of a flexible functionalized graphene hydrogel (FGH) thin film electrode; (b) low- and (c) high-magnification SEM images of interior microstructures of the FGH film; (d) digital photograph of a FGH-based flexible solid-state supercapacitor; (e) a schematic diagram of the solid-state device with $\mathrm{H}_{2} \mathrm{SO}_{4}$-PVA polymer gel as the electrolyte and separator. PVA: polyvinyl alcohol
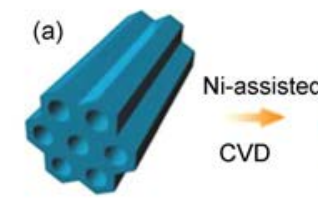

mesoporous $\mathrm{SiO}_{2}$

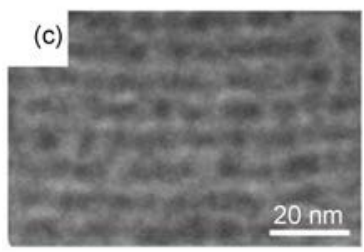

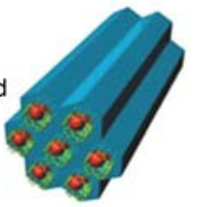

mesoporous $\mathrm{SiO}_{2}$ I $\mathrm{Ni} /$ few-layer carbon

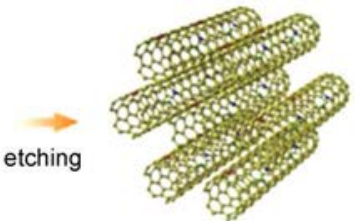

ordered mesoporous few-layer carbon (b)

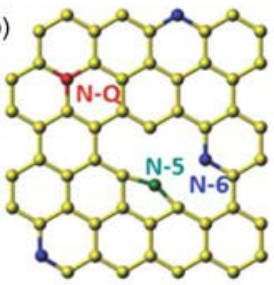

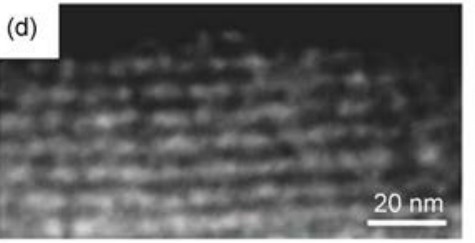

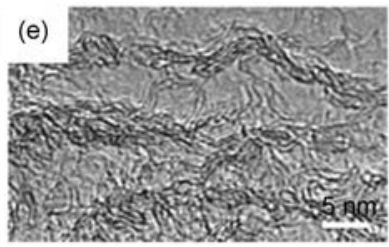

图 10 氮掺杂的有序介孔石墨烯及其相关材料的结构图 ${ }^{83}$

Fig.10 Structures of $\mathbf{N}$-doped ordered mesoporous few-layer carbon and related materials ${ }^{83}$

(a) fabrication schematic of ordered mesoporous few-layer carbon (OMFLC); (b) possible locations for $\mathrm{N}$ incorporation into a few-layer carbon network; high-angle annular dark-field transmission electron microscopy (TEM) images of (c) ordered mesoporous carbon (OMC) and (d) OMFLC; (e) high-resolution TEM image of OMFLC

低的电导率。

Huang 等 ${ }^{22,93}$ 制备了一种三维的 $\mathrm{CNTs} @ \mathrm{MnO}_{2}$ 核-壳纳米结构材料, 直接生长在 $\mathrm{Ni}$ 泡沫上, 不使 用任何粘结剂即可直接作为电极材料。如图 11 所 示, CNTs 直接生长在 $\mathrm{Ni}$ 泡沫上, 超薄的片状 $\mathrm{MnO}_{2}$ 均匀地包覆在 CNTs 表面。这种不使用粘结 剂的电极材料具有较高的比容量 (电流密度为 $0.3 \mathrm{~A}$. $\mathrm{g}^{-1}$ 时的比容量为 $325.5 \mathrm{~F} \cdot \mathrm{g}^{-1}$ )、良好的倍率性能以 及优异的循环稳定性(5000 次循环后的容量损失为 $9.5 \%)$ 。但是, 在发生氧化还原反应过程中碳材料
的消耗会引起CNTs 结构的坍塌, 导致电导率下降。

Shi 等 94.95 发明了一种新的方法, 利用 $\mathrm{N}$ 掺杂的 活性炭层作为牺牲材料制备 CNTs, 这种材料可以 负载相当大量的 $\mathrm{MnO}_{2}$ 。该方法充分发挥了小尺寸 $\mathrm{MnO}_{2}$ 纳米颗粒的优势, 可以与多壁碳纳米管以及 材料的纤维微结构具有良好的接触。电化学测试 结果显示, 当 $\mathrm{MnO}_{2}$ 负载量为 $20 \mathrm{mg} \cdot \mathrm{cm}^{-2}$ 时材料的 比容量达到 $6.29 \mathrm{~F} \cdot \mathrm{cm}^{-2}\left(311.7 \mathrm{~F} \cdot \mathrm{g}^{-1}\right)$, 并且循环稳 定性及在高充放电速率下电容保持性能良好。

石墨烯也是经常用来与 $\mathrm{MnO}_{2}$ 复合的碳材料。 

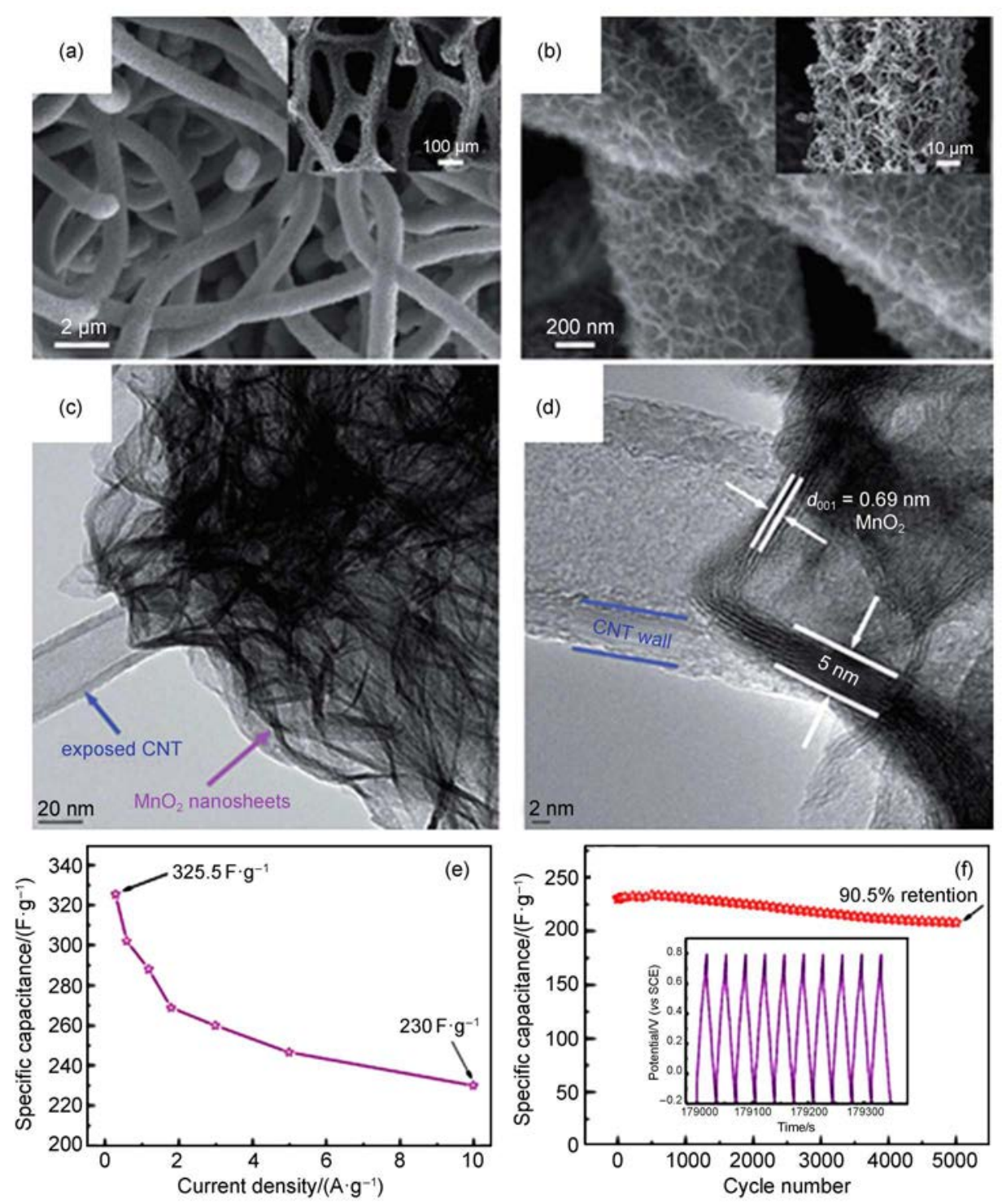

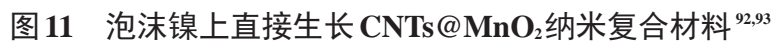

Fig.11 CNTs@ $\mathrm{MnO}_{2}$ nanocomposites directly grown on nickel foam ${ }^{92,93}$

(a, b) scanning electron microscopy (SEM) images of CNTs@ $\mathrm{MnO}_{2}$ core-shell nanostructures grown on nickel foam, insets show the corresponding local 3D structures in low magnifications; (c, d) TEM and HRTEM images of a partial coverage of the $\mathrm{MnO}_{2}$ nanosheets on the surface of CNTs;

(e, f) electrochemical properties of the as-prepared CNTs@ $\mathrm{MnO}_{2}$ core-shell nanocomposite electrode

例如, Cheng 等 ${ }^{23}$ 利用溶液相中石墨烯片和 $\mathrm{MnO}_{2}$ 纳米线的组装制备了石墨烯纳米片 $/ \mathrm{MnO}_{2}$ 纳米线的 复合物(图 12(a))。Liu 等 ${ }^{96}$ 通过一步法制备了石墨 烯纳米带和 $\mathrm{MnO}_{2}$ 纳米颗粒的新型复合材料 $(\mathrm{GNR}-$ $\left.\mathrm{MnO}_{2}\right)$ 。通过调节氧化剂 $\left(\mathrm{KMnO}_{4}\right)$ 的在合成过程中 的使用量，不仅可以使 CNT 形成 GNR，而且还可 以使 $\mathrm{MnO}_{2}$ 纳米颗粒可控的沉积 GNRs 的表面或中 间层上 (图 12(b))。将 GNR- $\mathrm{MnO}_{2}$ 复合材料、纯 GNR 纳米片分别作为非对称超电容器的正极和负 极, 具有宽的工作电势 $(2.0 \mathrm{~V})$ 和 $212 \mathrm{~F} \cdot \mathrm{g}^{-1}$ 的比电容 量。Song 等 ${ }^{97}$ 将 $\mathrm{MnO}_{2}$ 纳米片沉积在功能化的剥离
石墨烯基底上 $\left(\mathrm{FEG} / \mathrm{MnO}_{2}\right)$ (图 12(c))。功能化的超薄 石墨烯片层为 $\mathrm{MnO}_{2}$ 纳米片的沉积提供了大的导电 基底。 $\mathrm{FEG} / \mathrm{MnO}_{2}$ 作为电极材料的面积比容量为 $244 \mathrm{mF} \cdot \mathrm{cm}^{-2}, 5000$ 次循环后仍有 $92 \%$ 的容量存 留。 $\mathrm{Li}^{\text {等 }}{ }^{8}$ 报道了一种简便的方法, 将预合成的超 薄 $\beta-\mathrm{MnO}_{2}$ 纳米带分散在氧化石墨烯的前驱体溶液 中进行水热处理, 制备多级次和相互连通的还原氧 化石墨烯 $(\mathrm{rGO}) / \beta-\mathrm{MnO}_{2}$ 纳米带混合的水凝胶 (图 12 (d))。得到的 $\mathrm{rGO} / \beta-\mathrm{MnO}_{2}$ 纳米带混合凝胶中超薄 $\beta$ $\mathrm{MnO}_{2}$ 纳米带的含量达到 $54.2 \%$, 在电流密度为 1.0 $\mathrm{A} \cdot \mathrm{g}^{-1}$ 时的比容量为 $362 \mathrm{~F} \cdot \mathrm{g}^{-1}$, 循环稳定性良好。 

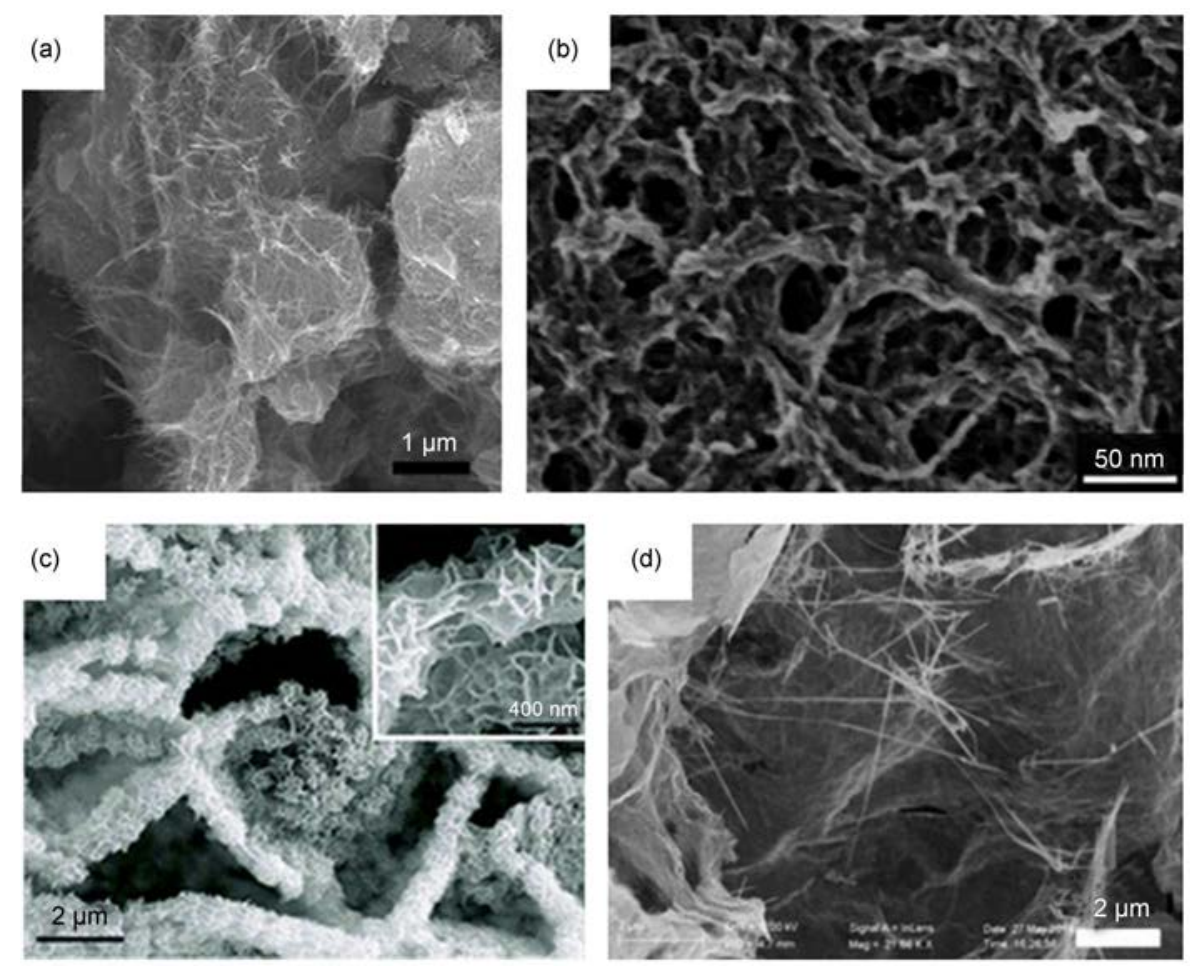

图 12 不同结构的 $\mathrm{GO} / \mathrm{MnO}_{2}$ 复合材料的 $\mathrm{SEM}$ 图

Fig.12 SEM images of $\mathrm{MnO}_{2}$-graphene composites with different structures

(a) graphene nanosheets $/ \mathrm{MnO}_{2}{ }^{23}$; (b) graphene nanoribbons $/ \mathrm{MnO}_{2}{ }^{94-96}$; (c) partially exfoliated graphite $/ \mathrm{MnO}_{2}{ }^{97}$; (d) $3 \mathrm{D}$ graphene hydrogel/ $\mathrm{MnO}_{2}{ }^{98}$

\subsubsection{3 其他金属氧化物-碳材料}

本课题组发展了一种三维石墨烯气凝胶-金属 氧化物纳米粒子复合材料(简称 GA/MO) 的通用制 备方法, 制备了负载氧化钛 $\left(\mathrm{TiO}_{2}\right)$ 、氧化铈 $\left(\mathrm{CeO}_{2}\right)$ 、氧化铁 $\left(\alpha-\mathrm{Fe}_{2} \mathrm{O}_{3}\right)$ 、四氧化三锰 $\left(\mathrm{Mn}_{3} \mathrm{O}_{4}\right)$ 氧化 物纳米粒子的石墨烯气凝胶, 并研究了石墨烯气 凝胶-氧化钛纳米粒子复合物(简称 $\mathrm{GA} / \mathrm{TiO}_{2}$ ) 在电容 脱盐领域的应用, 证明了石墨烯气凝胶的三维贯 穿网络结构, 以及与 $\mathrm{TiO}_{2}$ 纳米粒子的复合大大提 高了复合体系电容脱盐的吸附量、吸脱附速率和 循环性能 ${ }^{99}$ 。在 $100 \mathrm{mV} \cdot \mathrm{s}^{-1}$ 的电极扫描速率下 $\mathrm{GA} /$ $\mathrm{TiO}_{2}$ 的质量比电容为 $119.7 \mathrm{~F} \cdot \mathrm{g}^{-1}$, 远高于 $\mathrm{GA} 53.1$ $\mathrm{F} \cdot \mathrm{g}^{-1}$ 和 $\mathrm{AC}$ 的 $11.3 \mathrm{~F} \cdot \mathrm{g}^{-1}$, 且 $\mathrm{GA} / \mathrm{TiO}_{2}$ 具有很高的电 化学稳定性, 经过 1000 次循环伏安循环后 $\mathrm{GA} / \mathrm{TiO}_{2}$ 的电容未有任何变化。除了与电容脱盐相关的 0.1 $\mathrm{mol} \cdot \mathrm{L}^{-1} \mathrm{NaCl}$ 水溶液体系, $\mathrm{GA} / \mathrm{TiO}_{2}$ 在 $6 \mathrm{~mol} \cdot \mathrm{L}^{-1}$ $\mathrm{KOH}$ 中的质量比电容如图 13 所示。在 $6 \mathrm{~mol} \cdot \mathrm{L}^{-1}$ $\mathrm{KOH}$ 中, $\mathrm{GA} / \mathrm{TiO}_{2}$ 的质量比电容可达 $245.4 \mathrm{~F} \cdot \mathrm{g}^{-1}$ $\left(5 \mathrm{mV} \cdot \mathrm{s}^{-1}\right)$ 和 $152.5 \mathrm{~F} \cdot \mathrm{g}^{-1}\left(1000 \mathrm{mV} \cdot \mathrm{s}^{-1}\right)$ 。

Ramaprabhu等 ${ }^{100}$ 利用化学还原的方法合成了 一系列的 CNTs/金属氧化物复合材料, 包括 CNTs 复合 $\mathrm{RuO}_{2} 、 \mathrm{TiO}_{2} 、 \mathrm{SnO}_{2}$, 用作超级电容器的电极
材料。由于金属氧化物纳米晶体与功能化的 MWNT 复合后对电极材料提供了噟电容, 与单纯 的 MWNT 电极相比, $\mathrm{RuO}_{2} 、 \mathrm{TiO}_{2} 、 \mathrm{SnO}_{2}$ 与 MWNT 复合的电极材料的比容量有了显著的提高。

\subsection{2 碳材料-导电聚合物}

导电聚合物作为赝电容材料, 具有电荷密度 较高、成本较低等优点, 有可能制备出低等效串 联电阻和高功率的电化学装置。尽管作为噟电容 材料, 导电聚合物在酸性介质中的比容量高达 400-600 F· $\mathrm{g}^{-1}$, 但循环稳定性的问题严重阻碍了 导电聚合物作为超级电容器电极材料在实际中的 应用 ${ }^{101}$ 。对此普遍被接受的原因主要是由于导电聚 合物不稳定的化学结构和低的电荷转移。一般 地, 聚合物的结构在充电和放电过程中会发生膨 胀和皱缩, 导致导电聚合物的循环不稳定性。另 外, 块状的导电性聚合物具有比较紧密堆积的结 构, 会阻止电解质离子的渗透, 从而降低性能。 因此, 通常将导电聚合物与其它材料进行复合使 用。基于导电聚合物的纳米复合材料的电化学活 性、机械强度和导电性等都与导电聚合物固有的 性质及各组分的协同作用有关，目前的研究兴趣 主要集中在导电聚合物与几类重要的材料体系进 

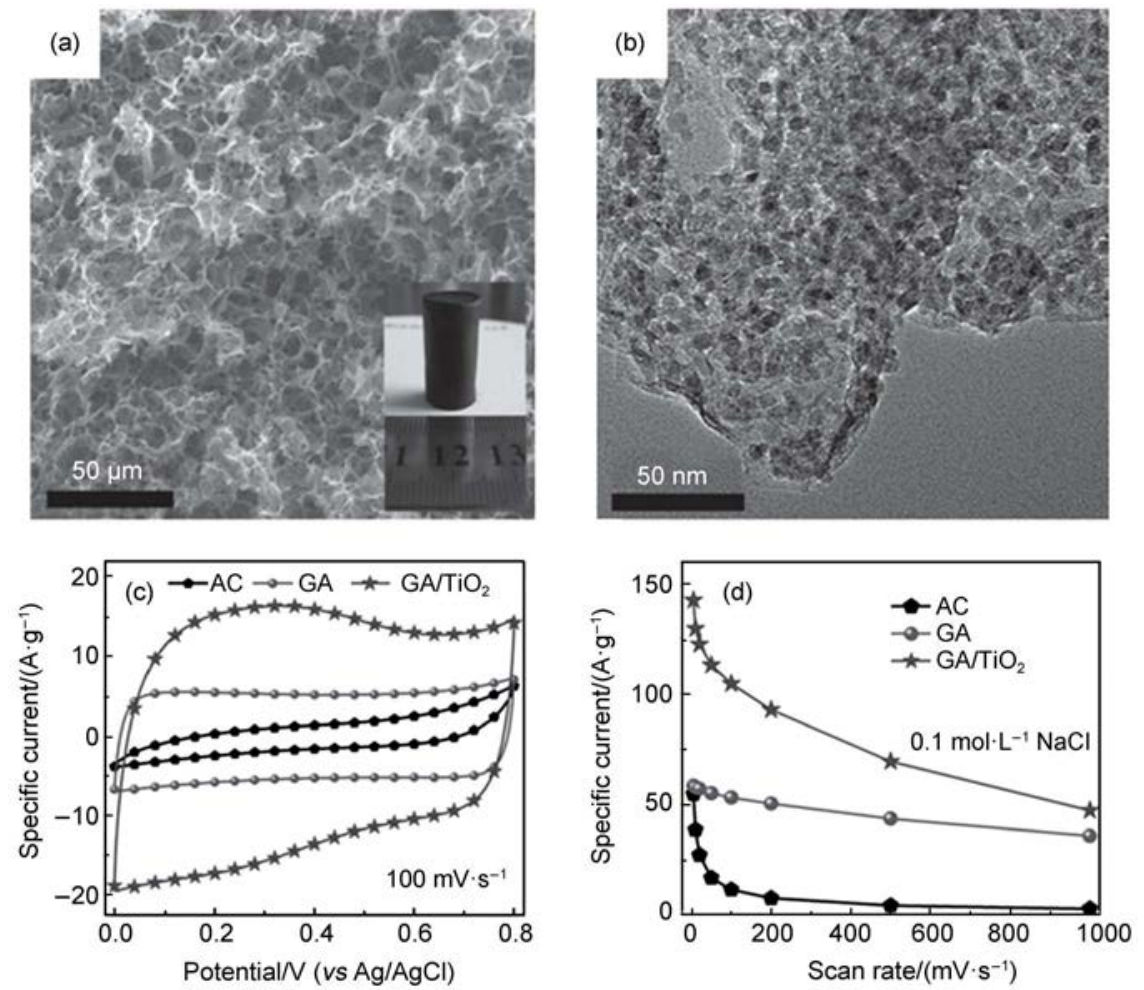

图 13 三维的石墨烯气凝胶-氧化钛纳米粒子复合物 ${ }^{99}$

Fig.13 3D GA/TiO ${ }_{2}$ nanocomposites ${ }^{99}$

(a) $\mathrm{SEM}$ images of $3 \mathrm{D} \mathrm{GA} / \mathrm{TiO}_{2}$ hybrids, inset in (a) is the image of the monolithic $\mathrm{GA} / \mathrm{TiO}_{2}$ product after freeze-drying;

(b) TEM image of GA/TiO 2 hybrids; (c) $\mathrm{CV}$ curves of $\mathrm{AC}, \mathrm{GA}, \mathrm{GA} / \mathrm{TiO}_{2}$ in $0.1 \mathrm{~mol} \cdot \mathrm{L}^{-1} \mathrm{NaCl}$ solution at a scan rate of $100 \mathrm{mV} \cdot \mathrm{s}^{-1}$; (d) the specific capacitance of $\mathrm{AC}, \mathrm{GA}, \mathrm{GA} / \mathrm{TiO}_{2}$ at different scan rates

行复合, 包括纤维素及其衍生物、碳纳米材料、 金属氧化物以及其它功能性材料。在此重点介绍 导电聚合物与碳材料的复合。

与导电聚合物复合的碳纳米材料主要是碳纳 米管、碳纳米纤维、石墨烯及活性炭等, 碳纳米 材料作为导电性骨架有利于电荷载流子的传输, 同时起到有助于导电聚合物在电化学过程中维持 结构的关键作用。单壁碳纳米管与聚苯胺的复合 物(SWCNT-PANI) 由一个典型的引发剂辅助聚合方 法合成, 从而得到 PANI 为纳米纤维壳和 SWCNT 为核心的纳米结构 ${ }^{102}$ 。类似地, 通过悬浮聚合的方 法在 RGO 的悬浮液中制备得到了聚苯胺-氧化石墨 烯(PANI-RGO) 的复合材料 ${ }^{103}$ 。

\subsection{3 其它二元复合体系}

本课题组将聚吡咯(PPy) 可控地生长在单层 $\mathrm{MoS}_{2}$ 片层上, 获得了高性能的超级电容器电极材 料, 如图 14 所示 ${ }^{104}$ 。这种新型纳米材料具有许多 特有的优势：1T相单层 $\mathrm{MoS}_{2}$ 片层的金属性质提供 高的导电性; PPy 与单层 $\mathrm{MoS}_{2}$ 片层表面强的相互 作用使 PPy 在单层 $\mathrm{MoS}_{2}$ 片上形成均一的超薄层,
从而在充放电过程中为电荷传输提供大的电化学 活性表面积和短的离子扩散长度; PPy 与 $\mathrm{MoS}_{2}$ 之 间的强相互作用提供了优异的界面接触, 从而有 效提高了超级电容器的循环稳定性等, 这些特点 都有利于电解质和电子的传输从而有效地存储电 荷。所以得到的 $\mathrm{MoS}_{2} / \mathrm{PPy}$ 纳米复合材料与之前研 究的所有导电聚合物基电极材料相比具有更高的 比容量、卓越的速率性能以及更好的循环稳定 性。以 $1 \mathrm{~mol} \cdot \mathrm{L}^{-1} \mathrm{KCl}$ 作为电解液, 工作电压为 $0.0-0.9 \mathrm{~V}$, 在扫描速率为 $10 \mathrm{mV} \cdot \mathrm{s}^{-1}$ 时的比容量约 为 $700 \mathrm{~F} \cdot \mathrm{g}^{-1}$, 最高能量密度达 $83.3 \mathrm{Wh} \cdot \mathrm{kg}^{-1}$ 。

$\mathrm{Yi}$ 等 ${ }^{105}$ 用化学浴沉积方法得到的 CNT@Ni $(\mathrm{OH})_{2}$ 核-壳结构复合物做阳极, 三维的石墨烯凝胶 (3DGN) 做阴极, 水系的 $\mathrm{KOH}$ 溶液做电解液制备非 对称超级电容器。三电极体系下测试单电极的电 容器性能优越, $\mathrm{CNT} @ \mathrm{Ni}(\mathrm{OH})_{2}$ 电极在电势窗口为 0-0.5 V 下电流密度为 $2 \mathrm{~A} \cdot \mathrm{g}^{-1}$ 时对应的比容量达到 $1136 \mathrm{~F} \cdot \mathrm{g}^{-1}$, 三维的石墨烯凝胶电极在电势窗口 为 $-1-0 \mathrm{~V}$ 下电流密度为 $1 \mathrm{~A} \cdot \mathrm{g}^{-1}$ 时对应的比容量 为 $203 \mathrm{~F} \cdot \mathrm{g}^{-1}$ 。基于以上单电极材料优良的性能, 


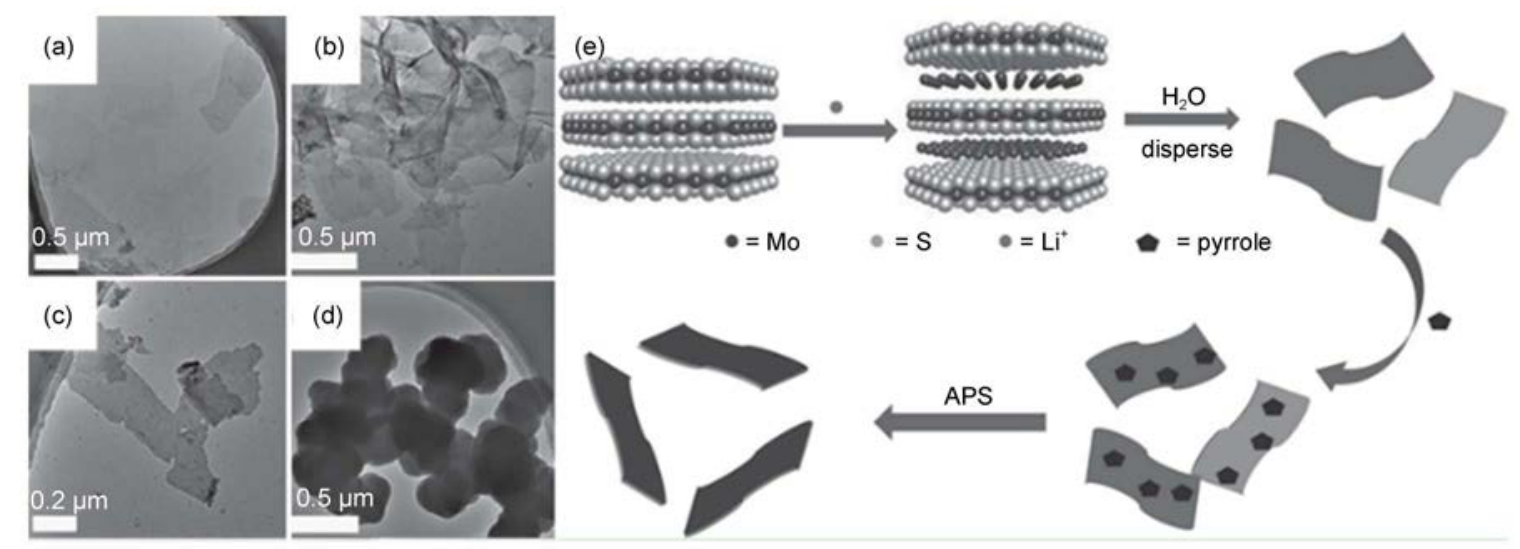

图 $14 \mathrm{MoS}_{2} / \mathrm{PPy}-\boldsymbol{n}$ 纳米复合材料 ${ }^{104}$

Fig.14 $\mathrm{MoS}_{2} / \mathrm{PPy}$ - $\boldsymbol{n}$ nanocomposites ${ }^{104}$

TEM images of (a) as-exfoliated $\mathrm{MoS}_{2}$ monolayers; (b, c) MoS 2 /PPy-2 nanocomposites; (d) pure polypyrrole; (e) fabrication process of $\mathrm{MoS}_{2} / \mathrm{PPy}-n$ nanocomposites

电压范围 0-1.6 V, 所组成的 CNT @ Ni(OH $)_{2} / / 3 D G N$ 非对称超级电容器在功率密度为 $800 \mathrm{~W} \cdot \mathrm{kg}^{-1}$ 时表现 出的最大的能量密度为 $44.0 \mathrm{Wh} \cdot \mathrm{kg}^{-1}$, 即使功率密度 高达 $16000 \mathrm{~W} \cdot \mathrm{kg}^{-1}$ 能量密度仍能保留 $19.6 \mathrm{Wh} \cdot \mathrm{kg}^{-1}$ 。

$\mathrm{Su}$ 等 ${ }^{106}$ 用连续的两步电化学沉积法制备出无 定型 $\mathrm{Ni}(\mathrm{OH})_{2} @ \mathrm{Ni}$ 核壳结构的电极材料用于高性能 的法拉第噟电容器。无定型的 $\mathrm{Ni}(\mathrm{OH})_{2}$ 直接生长在 $3 \mathrm{DNi}$ 纳米颗粒的表面, 可以为快速的电子传输提 供大量的传递通道, 而且使用电沉积的方法制备 的电极不需要外加任何粘结剂或炭黑等, 能有效 降低材料的内部电阻, 提高速率性能。电化学性 能研究表明无定型 $\mathrm{Ni}(\mathrm{OH})_{2} @ \mathrm{Ni}$ 核壳结构的电极材 料在 $1 \mathrm{mV} \cdot \mathrm{s}^{-1}$ 的扫描速率下比容量高达 $2868 \mathrm{~F}$. $\mathrm{g}^{-1}$, 即使在 $50 \mathrm{~A} \cdot \mathrm{g}^{-1}$ 这么高的充放电速率下的比容 量仍有 $1710 \mathrm{~F} \cdot \mathrm{g}^{-1}$, 同时材料也表现出很好的循环 稳定性。最近, 同课题组的 Chen 等 ${ }^{107}$ 以三维的纳 米金属片作为集流体, 在其表面电化学聚合PPy制 备核壳结构的PPy@3DNi 复合材料作为电极材 料, 用来提高 PPy 的电化学性能以及电极材料的利 用率。用作超级电容器电极材料同样获得了高比 容量和出色的循环稳定性。

除此之外, 两种碳材料进行复合也能获得良 好性能的超级电容器电极材料。Jiang 课题组 ${ }^{108}$ 利 用木耳吸水膨胀的特性, 将其浸渍到 $\mathrm{GO}$ 的水溶液 中得到木耳/GO 复合物, 水热法进行碳化后用 $\mathrm{KOH}$ 活化, 最终制得多孔碳/ $\mathrm{GO}$ 的纳米复合物。 这种材料可以实现介孔/微孔的平衡比例调控, 提 高材料的导电性, 同时具有高表面积、大的孔体 积。其作为电极材料组装成对称超级电容器具有
优异的性能, 在 $1 \mathrm{~mol} \cdot \mathrm{L}^{-1} \mathrm{H}_{2} \mathrm{SO}_{4}$ 水溶液中的比容 量达 $256 \mathrm{~F} \cdot \mathrm{g}^{-1}$, 极好的倍率特性以及稳定的循环 性能, 经 10000 次循环后的容量损失仅为 $8 \%$ 。此 外, 该课题组还将有序介孔碳与 CNTs 进行复合制 备三维纳米复合材料, 用作超级电容器的电极材 料, 同样具有很好的电容性能 ${ }^{109}$ 。

\section{3 三元体系复合电极}

将两种电极材料进行复合, 在一定程度上即 弥补了单一材料的缺点, 又可以保留各组分的优 点, 但仍存在一些问题。比如, 金属氧化物电极 材料与各种碳材料进行复合, 虽有助于改善其导 电性, 但不能完全解决其在充放电过程中发生的 晶格体积膨胀和收缩现象, 而且在充放电循环过 程中金属氧化物活性组分容易从碳材料表面脱 离, 导致循环稳定性降低, 在碳材料表面构筑金 属氧化物层后会显著降低电极材料的表面导电 性。基于以上, 近年来又发展了一种新型的设计 思路, 在二元复合材料的基础上引入第三种活性 组分, 利用各组分之间的协同作用进一步优化材 料的微观结构和结构稳定性。

Hou 等 ${ }^{110}$ 用一种简单且低成本的方法整合 $\mathrm{MnO}_{2} 、 \mathrm{CNT}$ 、导电聚合物三种材料设计合成了三 元纳米复合材料 $\mathrm{MnO}_{2} / \mathrm{CNT} / \mathrm{PEDOT}-\mathrm{PSS}$, 如图 15 $(a, b)$ 所示。在此复合物中, 对于提高能量存储中 金属氧化物的有效利用率, 各组分都起到了至关 重要的作用: CNTs 不仅为多孔 $\mathrm{MnO}_{2}$ 纳米微球的 沉积提供了高的表面积, 还能提高材料的导电性 以及机械稳定性; 多孔的 $\mathrm{MnO}_{2}$ 纳米微球的高比表 面积有利于比容量的提高; PEDOT-PSS 既可以作 

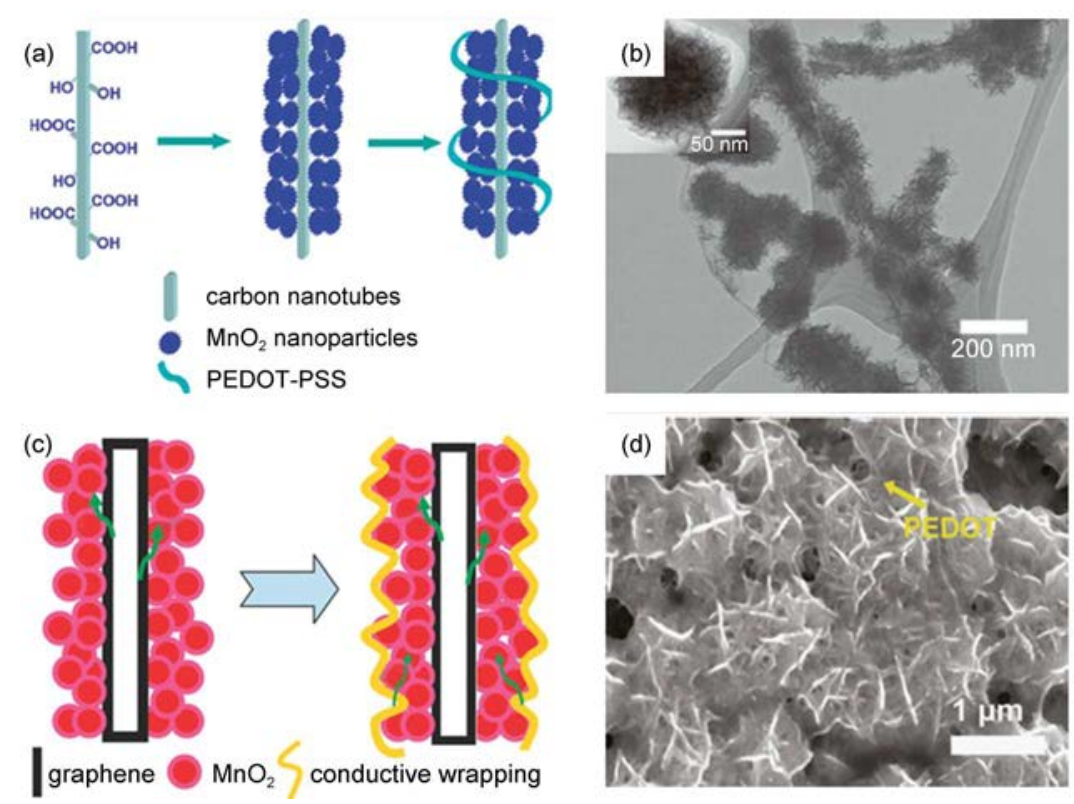

图 15 碳材料/金属氧化物/导电聚合物三元复合电极材料的结构示意图

Fig.15 Scheme of hybrid ternary electrodes based on carbon/metal oxides/conducting polymers

(a) schematic of $\mathrm{MnO}_{2} / \mathrm{CNTs} / \mathrm{PEDOT}-\mathrm{PSS}$ ternary composite material, (b) TEM of PEDOT-PSS dispersed $\mathrm{MnO}_{2}$ nano spheres in situ grown on $\mathrm{CNTs} ;{ }^{110}$ (c) schematic illustration showing the conductive wrapping of graphene/ $\mathrm{MnO}_{2}(\mathrm{GM})$ to introduce an additional electron transport path, (d) typical SEM image showing graphene/ $\mathrm{MnO}_{2} / \mathrm{PEDOT}$ :PSS nanostructures $(\mathrm{GMP})^{111}$.

PEDOT-PSS: poly(3,4-ethylenedioxythiophene)-polystrene sulfonate

为形成 $\mathrm{MnO}_{2} / \mathrm{CNT}$ 结构的有效分散剂, 又可以作为 粘结剂材料提高与基底的粘附力以及增强 $\mathrm{MnO}_{2} /$ CNT 颗粒之间的接触。将这三种材料组合在一起得 到的材料具有多孔的相互贯通的网络结构, 从而提 供高的比容量、倍率性能以及好的循环稳定性。比 容量最高可达 $427 \mathrm{~F} \cdot \mathrm{g}^{-1}$, 即使在 $\mathrm{MnO}_{2}$ 负载量达到 $60 \%$ 时仍能达到 $200 \mathrm{~F} \cdot \mathrm{g}^{-1}$ 。在循环稳定性测试中, 经过 1000 次循环后比容量仍然维持在 $99 \%$ 以上。

$\mathrm{Yu}$ 等 ${ }^{111}$ 发展了一种 “导电包皮” 的方法来提 高石墨烯 $/ \mathrm{MnO}_{2}$ 基纳米结构电极材料的超级电容器 性能, 如图 15(c, d) 所示。通过 CNTs 或导电聚合物 在石墨烯 $/ \mathrm{MnO}_{2}$ 基纳米结构电极表面形成 $3 \mathrm{D}$ 的导 电包皮, 这样制得的复合材料电极, 其比容量约 为 $380 \mathrm{~F} \cdot \mathrm{g}^{-1}$, 分别提高了 $20 \%$ 和 $40 \%$ 。此外这种 三元的复合电极循环性能稳定, 经过 3000 次循环 后的容量存留 $>95 \%$ 。折中 $3 \mathrm{D}$ 的导电包皮的方法 提供了一个增强金属氧化物基电化学超级电容器 器件性能的新的研究方向, 有助于设计下一代高 性能能量存储装置。基于上述工作, 表明通过简 单的浸渍工艺将额外的导电材料(碳纳米管或导电 聚合物)引入体系可以增强混合电极的电化学性能。

除了将碳材料、金属氧化物与导电聚合物这 三种材料进行复合外, 目前三元复合超级电容器
电极材料的研究还包括一种金属氧化物与两种不 同碳材料之间的复合以及两种金属氧化物与一种 碳材料的复合等。

Cheng 等 ${ }^{17}$ 用超声-过滤结合的方法制造了一种 基于石墨烯 $/ \mathrm{MnO}_{2} / \mathrm{CNTs}$ 纳米复合材料的高度灵 活、强韧、高导电性的薄膜, 用作超级电容器的 电极材料, 如图 16 所示。石墨烯、CNTs 和 $\mathrm{MnO}_{2}$ 的协同作用给材料提供了三种单独材料都无法实 现的优异的机械性能(48 MPa 的拉伸强度)和优越的 电化学活性。这种柔性电极允许高的活性材料负 载量 $\left(71 \%(w)\right.$ 的 $\left.\mathrm{MnO}_{2}\right)$, 电极制备过程中不需要使 用集流体和粘结剂。在三电极体系下测试, 具有 $372 \mathrm{~F} \cdot \mathrm{g}^{-1}$ 的高比容量和优异的倍率性能, 利用该 薄膜绕直径为 $0.5 \mathrm{~mm}$ 棒进行卷绕制造的全电池也 具有很高的超级电容器性能。 $\mathrm{Li}$ 等 ${ }^{112}$ 通过原位化学 聚合包覆法和水热法结合, 设计制备了一种多级 孔结构的CNTs@NCS@ $\mathrm{MnO}_{2}$ 核壳型复合物用作高 性能的超级电容器材料, 并以 CNTs@NCS@ $\mathrm{MnO}_{2}$ 和 $\mathrm{AC}$ 分别作为阳极和阴极组装成非对称超级电容 器。由于 $\mathrm{N}$ 掺杂的碳壳与 $\mathrm{MnO}_{2}$ 之间的协同作用和 良好的结构设计, CNTs@NCS@ $\mathrm{MnO}_{2}$ 电极展现出 较高的综合电化学性能: 高比电容、良好的倍率 性能、优越的可逆性和循环稳定性。 

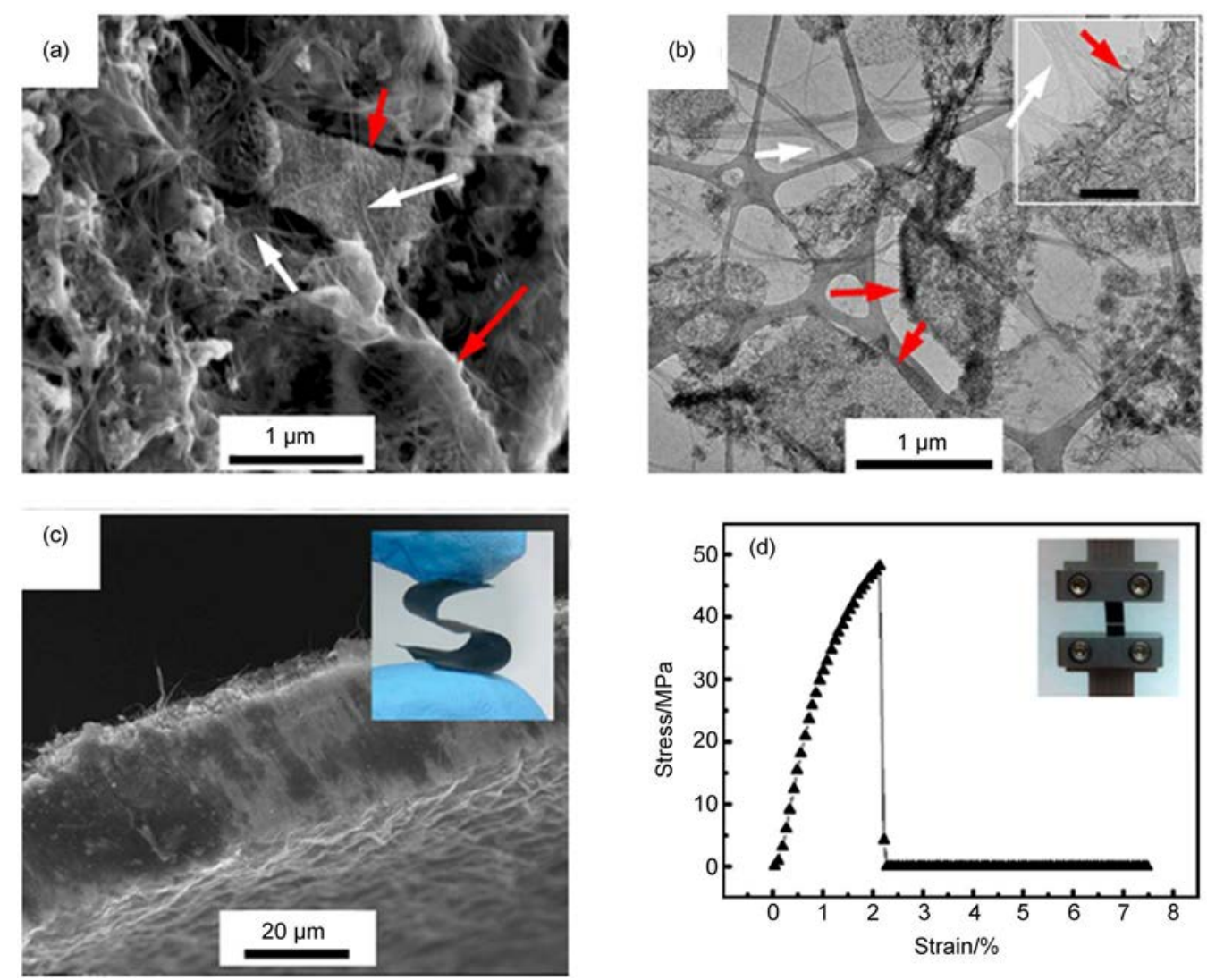

图 16 石墨烯/ $/ \mathrm{MnO}_{2} / \mathrm{CNTs}$ 三元复合材料制备薄膜电极 ${ }^{17}$

Fig.16 Hybrid ternary thin film electrode based on graphene/ $\mathrm{MnO}_{2} / \mathrm{CNTs}$ composites $^{17}$

(a) SEM and (b) TEM images of the interconnected structure formed by the graphene/ $\mathrm{MnO}_{2}$ composite (red arrows) and fFWNTs (white arrows);

(c) cross-sectional SEM image and a picture (inserted at the top right corner) of the film showing the flexibility of these structures;

(d) typical stress-strain curve for the graphene/ $\mathrm{MnO}_{2} / \mathrm{CNT}$ composite film with $25 \%(w)$ of fFWNTs. color online

Zhi 等 ${ }^{113}$ 构筑了一种一维银纳米线/三维石墨 烯/有序介孔碳复合材料, 该材料具有三维纳米导 电网络结构, 组装成柔性器件后, 电容性能优 异, 比容量达 $213 \mathrm{~F} \cdot \mathrm{g}^{-1}$, 且经 10000 次循环后的容 量仍能保持 $90 \%$ 以上。三维石墨烯材料具有高的导 电性、良好的机械强度和柔性; 高导电性一维银纳 米线的存在，可优化表面电子传输通道、减小内 阻。在该复合结构中，两种材料之间的协同作用使 有序介孔碳材料的电化学性能得到很大提高。

\section{4 结论与展望}

近年来纳米材料的广泛研究极大推动了超级 电容器等先进储能技术的蓬勃发展。但是, 较高 的成本及与传统的电池相比较低的能量密度, 使 其应用受到一定的限制。因此, 目前在高性能电 极材料研究方面尚有很多工作要做: (1) 复合化, 研究和合成二元、三元复合材料, 利用不同材料 间的协同作用弥补单一组分性能的不足; (2) 纳米
化, 纳米尺度下的材料比表面积高、表现出特殊 的物理化学性能, 具有 “快的电子传导、短的离 子输运” 的特点, 可大大缩短离子在电极材料中 的扩散距离, 从而大幅度增加电化学反应活性; (3) 建立更加合理的模拟分析方法, 进一步明确充 放电机理; (4) 发展新的工艺方法, 降低制造成 本, 提高材料的综合性能。在不牺牲材料的高功 率密度及周期寿命的前提下提高超级电容器的能 量密度。

我国对超级电容器的研究始于 20 世纪 80 年代 初, 虽然起步较晚, 但是随着中国政府不断加大 研究投入, 并把超级电容器关键材料及其器件设 计列入《国家中长期科学和技术发展规划纲要 (2006-2020年)》和《国家 “十一五” 科学技术发 展规划》, 经过多年来的不解努力, 目前已经取得 了很多重要的研究成果。展望未来, 我们有理由 相信随着新型高效的电极材料的不断开发和应 用, 以及相关理论研究的不断深入, 超级电容器 
的应用会更加成熟, 并在交通、通讯、医疗器 械、军事装备等诸多领域得到广泛的应用。

\section{References}

(1) Wang, G.; Zhang, L.; Zhang, J. Chem. Soc. Rev. 2012, 41, 797. doi: 10.1039/C1CS15060J

(2) Simon, P.; Gogotsi, Y. Nat. Mater. 2008, 7, 845. doi: 10.1038/nmat2297

(3) Chu, S.; Majumdar, A. Nature 2012, 488, 294. doi: 10.1038/ nature 11475

(4) Arico, A. S.; Bruce, P.; Scrosati, B.; Tarascon, J. M.; Van Schalkwijk, W. Nat. Mater. 2005, 4, 366. doi: 10.1038/ nmat 1368

(5) Yang, Z.; Zhang, J.; Kintner Meyer, M. C.; Lu, X.; Choi, D.; Lemmon, J. P.; Liu, J. Chem. Rev. 2011, 111, 3577. doi: $10.1021 / \mathrm{cr} 100290 \mathrm{v}$

(6) Winter, M.; Brodd, R. J. Chem. Rev. 2004, 104, 4245. doi: $10.1021 / \mathrm{cr} 020730 \mathrm{k}$

(7) Zhang, Y.; Gui, Y.; Wu, X.; Feng, H.; Zhang, A.; Wang, L.; Xia, T. Int. J. Hydrog. Energy 2009, 34, 2467. doi: 10.1016/j. ijhydene.2008.12.078

(8) Miller, J. R.; Simon, P. Science Magazine 2008, 321, 651. doi: $10.1126 /$ science. 1158736

(9) Service, R. F. Science 2006, 313, 902. doi: 10.1126/ science.313.5789.902

(10) Conway, B. Electrochemical Supercapacitors-Scientific Fundamentals and Technological Applications; Plenum Press: New York, 1999; pp 11-30.

(11) Long, J. W.; Bélanger, D.; Brousse, T.; Sugimoto, W.; Sassin, M. B.; Crosnier, O. Mrs. Bull. 2011, 36, 513. doi: 10.1557/ mrs.2011.137

(12) Yu, G.; Xie, X.; Pan, L.; Bao, Z.; Cui, Y. Nano Energy 2013, 2, 213. doi: 10.1016/j.nanoen.2012.10.006

(13) Bhattacharjya, D.; Yu, J. S. J. Power Sources 2014, 262, 224. doi: 10.1016/j.jpowsour.2014.03.143

(14) Yi, H.; Wang, H.; Jing, Y.; Peng, T.; Wang, X. J. Power Sources 2015, 285, 281. doi:10.1016/j.jpowsour.2015.03.106

(15) Fan, W.; Xia, Y. Y.; Tjiu, W. W.; Pallathadka, P. K.; He, C.; Liu, T. J. Power Sources 2013, 243, 973. doi: 10.1016/j. jpowsour.2013.05.184

(16) Wang, H.; Gao, Q.; Hu, J. Microporous Mesoporous Mat. 2010, 131, 89. doi: 10.1016/j.micromeso.2009.12.007

(17) Cheng, Y.; Lu, S.; Zhang, H.; Varanasi, C. V.; Liu, J. Nano Lett. 2012, 12, 4206. doi: 10.1021/n1301804c

(18) Soin, N.; Roy, S. S.; Mitra, S. K.; Thundat, T.; McLaughlin, J. A. J. Mater. Chem. 2012, 22, 14944. doi: 10.1039/ C2JM31226C

(19) Wang, B.; Chen, J. S.; Wang, Z.; Madhavi, S.; Lou, X. W. D. Adv. Eng. Mater. 2012, 2, 1188. doi: 10.1002/aenm.201200008

(20) Yuan, C.; Yang, L.; Hou, L.; Shen, L.; Zhang, X.; Lou, X. W.
D. Energy Environ. Sci. 2012, 5, 7883. doi: 10.1039/ C2EE21745G

(21) Naoi, K.; Morita, M. Electrochem. Soc. Interface 2008, 17, 44.

(22) Naoi, K.; Simon, P. Electrochem. Soc. Interface 2008, 17, 34.

(23) Wu, Z. S.; Ren, W.; Wang, D. W.; Li, F.; Liu, B.; Cheng, H. M. ACS Nano 2010, 4, 5835. doi: 10.1021/nn101754k

(24) Sivakkumar, S.; Kim, W. J.; Choi, J. A.; MacFarlane, D. R.; Forsyth, M.; Kim, D. W. J. Power Sources 2007, 171, 1062. doi: 10.1016/j.jpowsour.2007.05.103

(25) Chen, P. C.; Shen, G.; Shi, Y.; Chen, H.; Zhou, C. ACS Nano 2010, 4, 4403. doi: 10.1021/nn100856y

(26) Yan, J.; Wang, Q.; Wei, T.; Fan, Z. Adv. Eng. Mater. 2014, 4, 1300816. doi: 10.1002/aenm.201300816

(27) Wei, L.; Sevilla, M.; Fuertes, A. B.; Mokaya, R.; Yushin, G. Adv. Eng. Mater. 2011, 1, 356. doi: 10.1002/aenm.201100019

(28) Yan, J.; Wei, T.; Qiao, W.; Fan, Z.; Zhang, L.; Li, T.; Zhao, Q. Electrochem. Commun. 2010, 12, 1279. doi: 10.1016/j. elecom.2010.06.037

(29) Wei, L.; Sevilla, M.; Fuertes, A. B.; Mokaya, R.; Yushin, G. Adv. Funct. Mater. 2012, 22, 827. doi: 10.1002/ adfm. 201101866

(30) Ania, C. O.; Khomenko, V.; Raymundo Piñero, E.; Parra, J. B.; Béguin, F. Adv. Funct. Mater. 2007, 17, 1828. doi: 10.1002/ adfm.200600961

(31) Zhou, H. C.; Long, J. R.; Yaghi, O. M. Chem. Rev. 2012, 112, 673. doi: $10.1021 / \mathrm{cr} 300014 \mathrm{x}$

(32) Sakata, Y.; Furukawa, S.; Kondo, M.; Hirai, K.; Horike, N.; Takashima, Y.; Uehara, H.; Louvain, N.; Meilikhov, M.; Tsuruoka, T. Science 2013, 339, 193. doi: 10.1126/ science. 1231451

(33) Tranchemontagne, D. J.; Mendoza Cortés, J. L.; O'Keeffe, M.; Yaghi, O. M. Chem. Soc. Rev. 2009, 38, 1257. doi: 10.1039/ B817735J

(34) Perry Iv, J. J.; Perman, J. A.; Zaworotko, M. J. Chem. Soc. Rev. 2009, 38, 1400. doi: 10.1039/B807086P

(35) O'Keeffe, M. Chem. Soc. Rev. 2009, 38, 1215. doi: 10.1039/ B802802H

(36) Furukawa, H.; Ko, N.; Go, Y. B.; Aratani, N.; Choi, S. B.; Choi, E.; Yazaydin, A. Ö.; Snurr, R. Q.; O'Keeffe, M.; Kim, J. Science 2010, 329, 424. doi: 10.1126/science.1192160

(37) Farha, O. K.; Yazaydın, A. Ö.; Eryazici, I.; Malliakas, C. D.; Hauser, B. G.; Kanatzidis, M. G.; Nguyen, S. T.; Snurr, R. Q.; Hupp, J. T. Nat. Chem. 2010, 2, 944. doi: 10.1038/nchem.834

(38) Farha, O. K.; Eryazici, I.; Jeong, N. C.; Hauser, B. G.; Wilmer, C. E.; Sarjeant, A. A.; Snurr, R. Q.; Nguyen, S. T.; Yazaydın, A. O. Z. R.; Hupp, J. T. J. Am. Chem. Soc. 2012, 134, 15016 doi: $10.1021 / \mathrm{ja} 3055639$

(39) Zhao, X.; Xiao, B.; Fletcher, A. J.; Thomas, K. M.; Bradshaw, D.; Rosseinsky, M. J. Science 2004, 306, 1012. doi: 10.1126/ science. 1101982

(40) Sumida, K.; Rogow, D. L.; Mason, J. A.; McDonald, T. M.; 
Bloch, E. D.; Herm, Z. R.; Bae, T. H.; Long, J. R. Chem. Rev. 2011, 112, 724. doi: 10.1021/cr2003272

(41) Yoon, M.; Srirambalaji, R.; Kim, K. Chem. Rev. 2011, 112, 1196. doi: $10.1021 / \mathrm{cr} 2003147$

(42) Lee, J.; Farha, O. K.; Roberts, J.; Scheidt, K. A.; Nguyen, S. T.; Hupp, J. T. Chem. Soc. Rev. 2009, 38, 1450. doi: 10.1039/ B807080F

(43) Yanai, N.; Kitayama, K.; Hijikata, Y.; Sato, H.; Matsuda, R.; Kubota, Y.; Takata, M.; Mizuno, M.; Uemura, T.; Kitagawa, S. Nat. Mater. 2011, 10, 787. doi: 10.1038/nmat3104

(44) Chen, B.; Yang, Y.; Zapata, F.; Lin, G.; Qian, G.; Lobkovsky, E. B. Adv. Mater. 2007, 19, 1693. doi: 10.1002/ adma.200601838

(45) Sadakiyo, M.; Ōkawa, H.; Shigematsu, A.; Ohba, M.; Yamada, T.; Kitagawa, H. J. Am. Chem. Soc. 2012, 134, 5472. doi: $10.1021 / \mathrm{ja} 300122 \mathrm{r}$

(46) Horcajada, P.; Chalati, T.; Serre, C.; Gillet, B.; Sebrie, C.; Baati, T.; Eubank, J. F.; Heurtaux, D.; Clayette, P.; Kreuz, C. Nature Mater. 2010, 9, 172. doi: 10.1038/nmat2608

(47) Liu, B.; Shioyama, H.; Akita, T.; Xu, Q. J. Am. Chem. Soc. 2008, 130, 5390. doi: 10.1021/ja7106146

(48) Li, S.L.; Xu, Q. Energy Environ. Sci. 2013, 6, 1656. doi: 10.1039/C3EE40507A

(49) Chaikittisilp, W.; Ariga, K.; Yamauchi, Y. J. Mater. Chem. A 2013, 1, 14. doi: 10.1039/C2TA00278G

(50) Liu, B.; Shioyama, H.; Jiang, H.; Zhang, X.; Xu, Q. Carbon 2010, 48, 456. doi: 10.1016/j.carbon.2009.09.061

(51) Jin, S. L.; Deng, H. G.; Liang, Z.; Qiao, W. M.; Ling, L. C. New Carbon Mater. 2012, 27, 87. doi: 10.1016/S1872-5805(12) 60005-5

(52) Hu, J.; Wang, H.; Gao, Q.; Guo, H. Carbon 2010, 48, 3599. doi: 10.1016/j.carbon.2010.06.008

(53) Jiang, H. L.; Liu, B.; Lan, Y. Q.; Kuratani, K.; Akita, T.; Shioyama, H.; Zong, F.; Xu, Q. J. Am. Chem. Soc. 2011, 133, 11854. doi: $10.1021 / \mathrm{ja} 203184 \mathrm{k}$

(54) Hu, M.; Reboul, J.; Furukawa, S.; Torad, N. L.; Ji, Q.; Srinivasu, P.; Ariga, K.; Kitagawa, S.; Yamauchi, Y. J. Am. Chem. Soc. 2012, 134, 2864. doi: 10.1021/ja208940u

(55) Tang, J.; Salunkhe, R. R.; Liu, J.; Torad, N. L.; Imura, M.; Furukawa, S.; Yamauchi, Y. J. Am. Chem. Soc. 2015, 137, 1572. doi: 10.1021/ja511539a

(56) Dresselhaus, M. S.; Dresselhaus, G.; Eklund, P. C. Science of Fullerenes and Carbon Nanotubes: Their Properties and Applications; Academic Press: San Diego, 1996; pp 757-870.

(57) Saito, R.; Dresselhaus, G.; Dresselhaus, M. J. Appl. Phys. 1993, 73, 494. doi: 10.1063/1.353358

(58) Issi, J. P.; Langer, L.; Heremans, J.; Olk, C. Carbon 1995, 33, 941. doi: 10.1016/0008-6223(95)00023-7

(59) Niu, C.; Sichel, E. K.; Hoch, R.; Moy, D.; Tennent, H. Appl. Phys. Lett. 1997, 70, 1480. doi: 10.1063/1.118568

(60) Ebbesen, T.; Lezec, H.; Hiura, H.; Bennett, J.; Ghaemi, H.;
Thio, T. Nature 1996, 382, 54. doi: 10.1038/382054a0

(61) Zhang, L. L.; Zhao, X. Chem. Soc. Rev. 2009, 38, 2520. doi: 10.1039/B813846J

(62) An, H. F.; Wang, X. Y.; Li, N.; Zheng, L. P.; Chen, Q. Q. Prog Chem. 2009, 21, 1832. [安红芳, 王先友, 李 娜, 郑丽萍, 陈 权启. 化学进展, 2009, 21, 1832.]

(63) Meng, F.; Ding, Y. Adv. Mater. 2011, 23, 4098. doi: 10.1002/ adma.201101678

(64) Fu, Y.; Cai, X.; Wu, H.; Lv, Z.; Hou, S.; Peng, M.; Yu, X.; Zou, D. Adv. Mater. 2012, 24, 5713. doi: 10.1002/adma.201202930

(65) Niu, Z.; Zhou, W.; Chen, J.; Feng, G.; Li, H.; Ma, W.; Li, J.; Dong, H.; Ren, Y.; Zhao, D. Energy Environ. Sci. 2011, 4, 1440. doi: 10.1039/C0EE00261E

(66) Kaempgen, M.; Chan, C. K.; Ma, J.; Cui, Y.; Gruner, G. Nano Lett. 2009, 9, 1872. doi: 10.1021/n18038579

(67) Hu, L.; Wu, H.; Cui, Y. Appl. Phys. Lett. 2010, 96, 183502. doi: $10.1063 / 1.3425767$

(68) Hu, L.; Pasta, M.; Mantia, F. L.; Cui, L.; Jeong, S.; Deshazer, H. D.; Choi, J. W.; Han, S. M.; Cui, Y. Nano Lett. 2010, 10, 708. doi: $10.1021 / \mathrm{n} 1903949 \mathrm{~m}$

(69) Brown, B.; Parker, C. B.; Stoner, B. R.; Grill, W. M.; Glass, J. T. J. Electrochem. Soc. 2011, 158, K217. doi: 10.1149/ 2.093112jes

(70) Zhang, H.; Cao, G.; Yang, Y. Energy Environ. Sci. 2009, 2, 932. doi: 10.1039/B906812K

(71) Jiang, H.; Ma, J.; Li, C. Adv. Mater. 2012, 24, 4197. doi: 10.1002/adma.201104942

(72) Novoselov, K. S.; Geim, A. K.; Morozov, S.; Jiang, D.; Zhang, Y.; Dubonos, S. A.; Grigorieva, I.; Firsov, A. Science 2004, 306, 666. doi: 10.1126/science. 1102896

(73) Allen, M. J.; Tung, V. C.; Kaner, R. B. Chem. Rev. 2009, 110, 132. doi: $10.1021 / \mathrm{cr} 900070 \mathrm{~d}$

(74) Rao, C. E. N. E. R.; Sood, A. E. K.; Subrahmanyam, K. E. S.; Govindaraj, A. Angew. Chem. Int. Edit. 2009, 48, 7752. doi: 10.1002/anie.200901678

(75) Chen, D.; Tang, L.; Li, J. Chem. Soc. Rev. 2010, 39, 3157. doi: 10.1039/B923596E

(76) Lee, C.; Wei, X.; Kysar, J. W.; Hone, J. Science 2008, 321, 385. doi: $10.1126 /$ science. 1157996

(77) Hu, C.; Song, L.; Zhang, Z.; Chen, N.; Feng, Z.; Qu, L. Energy Environ. Sci. 2015, 8, 31. doi: 10.1039/C4EE02594F

(78) Dai, L. Accounts Chem. Res. 2012, 46, 31. doi: 10.1021/ $\operatorname{ar} 300122 \mathrm{~m}$

(79) Wassei, J. K.; Kaner, R. B. Accounts Chem. Res. 2013, 46, 2244. doi: $10.1021 / \operatorname{ar} 300184 \mathrm{v}$

(80) Huang, X.; Zeng, Z.; Fan, Z.; Liu, J.; Zhang, H. Adv. Mater. 2012, 24, 5979. doi: 10.1002/adma.201201587

(81) Chen, P.; Yang, J. J.; Li, S. S.; Wang, Z.; Xiao, T. Y.; Qian, Y. H.; Yu, S. H. Nano Energy 2013, 2, 249. doi: 10.1016/j. nanoen.2012.09.003

(82) Xu, Y.; Lin, Z.; Huang, X.; Wang, Y.; Huang, Y.; Duan, X. Adv. 
Mater. 2013, 25, 5779. doi: 10.1002/adma.201301928

(83) Lin, T. Q.; Chen, I. W.; Liu, F. X.; Yang, C. Y.; Bi, H.; Xu, F. F.; Huang, F. Q. Science 2015, 350, 1508. doi: 10.1126/science. aab3798

(84) Conway, B.; Birss, V.; Wojtowicz, J. J. Power Sources 1997, 66, 1. doi: 10.1016/S0378-7753(96)02474-3

(85) Wang, X. F.; Wang, D. Z.; Liang, J. Rare Metal Mat. Eng. 2003, 32, 424. [王晓峰, 王大志, 梁 吉. 稀有金属材料与工 程, 2003, 32, 424.]

(86) Wu, Z. S.; Wang, D. W.; Ren, W.; Zhao, J.; Zhou, G.; Li, F.; Cheng, H. M. Adv. Funct. Mater. 2010, 20, 3595. doi: 10.1002/ adfm.201001054

(87) Hsieh, T. F.; Chuang, C. C.; Chen, W. J.; Huang, J. H.; Chen, W. T.; Shu, C. M. Carbon 2012, 50, 1740. doi: 10.1016/j. carbon.2011.12.017

(88) Bélanger, D.; Brousse, L.; Long, J. W. Electrochem. Soc. Interface 2008, 17, 49 .

(89) Jiang, H.; Zhao, T.; Ma, J.; Yan, C.; Li, C. Chem. Commun. 2011, 47, 1264. doi: 10.1039/C0CC04134C

(90) Toupin, M.; Brousse, T.; Bélanger, D. Chem. Mater. 2004, 16, 3184. doi: $10.1021 / \mathrm{cm} 049649 \mathrm{j}$

(91) Ghodbane, O.; Ataherian, F.; Wu, N. L.; Favier, F. J. Power Sources 2012, 206, 454. doi: 10.1016/j.jpowsour.2012.01.103

(92) Huang, M.; Li, F.; Dong, F.; Zhang, Y. X.; Zhang, L. L. J. Mater. Chem. A 2015, 3, 21380. doi: 10.1039/C5TA05523G

(93) Huang, M.; Mi, R.; Liu, H.; Li, F.; Zhao, X. L.; Zhang, W.; He, S. X.; Zhang, Y. X. J. Power Sources 2014, 269, 760. doi: 10.1016/j.jpowsour.2014.07.031

(94) Shi, K.; Zhitomirsky, I. ChemElectroChem. 2015, 2, 396. doi: 10.1002/celc.201402343

(95) Shi, K.; Ren, M.; Zhitomirsky, I. ACS Sustainable Chem. Eng. 2014, 2, 1289. doi: 10.1021/sc500118r

(96) Liu, M.; Tjiu, W. W.; Pan, J.; Zhang, C.; Gao, W.; Liu, T. Nanoscale 2014, 6, 4233. doi: 10.1039/C3NR06650A

(97) Song, Y.; Feng, D. Y.; Liu, T. Y.; Li, Y.; Liu, X. X. Nanoscale 2015, 7, 3581. doi: 10.1039/C4NR06559J

(98) Zhu, S.; Zhang, H.; Chen, P.; Nie, L. H.; Li, C. H.; Li, S. K. J.
Mater. Chem. A 2014, 3, 1540. doi: 10.1039/c4ta04921g

(99) Yin, H.; Zhao, S.; Wan, J.; Tang, H.; Chang, L.; He, L.; Zhao, H.; Gao, Y.; Tang, Z. Adv. Mater. 2013, 25, 6270. doi: 10.1002/ adma.201302223

(100) Reddy, A. L. M.; Ramaprabhu, S. J. Phys. Chem. C 2007, 111, 7727. doi: 10.1021/jp069006m

(101) Dubal, D. P.; Lee, S. H.; Kim, J. G.; Kim, W. B.; Lokhande, C. D. J. Mater. Chem. 2012, 22, 3044. doi: 10.1039/C2JM14470K

(102) Liao, Y.; Zhang, C.; Zhang, Y.; Strong, V.; Tang, J.; Li, X. G.; Kalantar Zadeh, K.; Hoek, E. M.; Wang, K. L.; Kaner, R. B. Nano Lett. 2011, 11, 954. doi: 10.1021/n1103322b

(103) Zhang, J.; Jiang, J.; Li, H.; Zhao, X. Energy Environ. Sci. 2011, 4, 4009. doi: 10.1039/C1EE01354H

(104) Tang, H.; Wang, J.; Yin, H.; Zhao, H.; Wang, D.; Tang, Z. Adv. Mater. 2015, 27, 1117. doi: 10.1002/adma.201404622

(105) Yi, H.; Wang, H.; Jing, Y.; Peng, T.; Wang, Y.; Guo, J.; He, Q.; Guo, Z.; Wang, X. J. Mater. Chem. A 2015, 3, 19545. doi: 10.1039/C5TA06174A

(106) Su, Y. Z.; Xiao, K.; Li, N.; Liu, Z. Q.; Qiao, S. Z. J. Mater. Chem. A 2014, 2, 13845. doi: 10.1039/C4TA02486A

(107) Chen, G. F.; Su, Y. Z.; Kuang, P. Y.; Liu, Z. Q.; Chen, D. Y.; Wu, X.; Li, N.; Qiao, S. Z. Chemistry 2015, 21, 4614. doi: 10.1002/chem.201405976

(108) Zhu, Z.; Jiang, H.; Guo, S.; Cheng, Q.; Hu, Y.; Li, C. Sci. Rep. 2015, 5, 15936. doi: 10.1038/srep15936

(109) Zhu, Z.; Hu, Y.; Jiang, H.; Li, C. J. Power Sources 2014, 246, 402. doi: 10.1016/j.jpowsour.2013.07.086

(110) Hou, Y.; Cheng, Y.; Hobson, T.; Liu, J. Nano Lett. 2010, 10, 2727. doi: $10.1021 / \mathrm{nl} 101723 \mathrm{~g}$

(111) Yu, G.; Hu, L.; Liu, N.; Wang, H.; Vosgueritchian, M.; Yang, Y.; Cui, Y.; Bao, Z. Nano Lett. 2011, 11, 4438. doi: 10.1021/ n12026635

(112) Li, L.; Li, R.; Gai, S.; Gao, P.; He, F.; Zhang, M.; Chen, Y.; Yang, P. J. Mater. Chem. A 2015, 3, 15642. doi: 10.1039/ C5TA03224E

(113) Zhi, J.; Zhao, W.; Liu, X.; Chen, A.; Liu, Z.; Huang, F. Adv. Funct. Mater. 2014, 24, 2013. doi: 10.1002/adfm.201303082 